\title{
Human feeding biomechanics: performance, variation, and functional constraints
}

Justin A. Ledogar ${ }^{\text {Corresp., }}{ }^{1,2}$ ， Paul C. Dechow ${ }^{3}$ ， Qian Wang ${ }^{4}$, Poorva H Gharpure ${ }^{4}$, Adam D. Gordon ${ }^{2}$, Karen L. Baab ${ }^{5}$, Amanda L. Smith ${ }^{2,6}$, Gerhard W. Weber ${ }^{7}$, Ian R. Grosse ${ }^{8}$, Callum F. Ross ${ }^{9}$, Brian G. Richmond ${ }^{10,11}$, Barth W. Wright ${ }^{12}$, Craig Byron ${ }^{13}$, Stephen Wroe ${ }^{14}$, David S. Strait ${ }^{2,6}$

1 Zoology Division, School of Environmental and Rural Science, University of New England, Armidale, New South Wales, Australia

2 Department of Anthropology, State University of New York at Albany, Albany, New York, United States

3 Department of Biomedical Sciences, Texas A\&M University Baylor College of Dentistry, Dallas, Texas, United States

4 Department of Biomedical Sciences, Baylor College of Dentistry, Dallas, Texas, United States

5 Department of Anatomy, Midwestern University, Glendale, Arizona, United States

6 Department of Anthropology, Washington University in St. Louis, St. Louis, Missouri, United States

7 Department of Anthropology, University of Vienna, Vienna, Austria

8 Department of Mechanical \& Industrial Engineering, University of Massachusetts, Amherst, Massachusetts, United States

9 Department of Organismal Biology \& Anatomy, University of Chicago, Chicago, Illinois, United States

10 Division of Anthropology, American Museum of Natural History, New York, New York, United States

11 Department of Human Evolution, Max Planck Institute for Evolutionary Anthropology, Leipzig, Germany

12 Department of Anatomy, Kansas City University of Medicine and Biosciences, Kansas City, Missouri, United States

13 Department of Biology, Mercer University, Macon, Georgia, United States

14 FEARlab, Zoology Division, School of Environmental and Rural Science, University of New England, Armidale, New South Wales, Australia

Corresponding Author: Justin A. Ledogar

Email address: JLedogar@gmail.com

The evolution of the modern human (Homo sapiens) cranium is characterized by a reduction in the size of the feeding system, including reductions in the size of the facial skeleton, postcanine teeth, and the muscles involved in biting and chewing. The conventional view hypothesizes that gracilization of the human feeding system is related to a shift toward eating foods that were less mechanically challenging to consume and/or foods that were processed using tools before being ingested. This hypothesis predicts that human feeding systems should not be well-configured to produce forceful bites and that the cranium should be structurally weak. An alternate hypothesis states that the modern human face is adapted to generate and withstand high biting forces. We used finite element analysis (FEA) to test two opposing mechanical hypotheses: that compared to our closest living relative, chimpanzees (Pan troglodytes), the modern human craniofacial skeleton is 1 ) less well configured, or 2) better configured to generate and withstand high magnitude bite forces. We considered intraspecific variation in our examination of human feeding biomechanics by examining a sample of geographically diverse crania that differed notably in shape. We found that our biomechanical models of human crania had broadly similar mechanical behavior despite their shape variation and were, on average, less 
structurally stiff than the crania of chimpanzees during unilateral biting when loaded with physiologically-scaled muscle loads. Our results also show that modern humans are efficient producers of bite force, consistent with previous analyses. However, highly tensile reaction forces were generated at the working (biting) side jaw joint during unilateral molar bites in which the chewing muscles were recruited with bilateral symmetry. In life, such a configuration would have increased the risk of joint dislocation and constrained the maximum recruitment levels of the masticatory muscles on the balancing (non-biting) side of the head. Our results do not necessarily conflict with the hypothesis that anterior tooth (incisors, canines, premolars) biting could have been selectively important in humans, although the reduced size of the premolars in humans has been shown to increase the risk of tooth crown fracture. We interpret our results to suggest that human craniofacial evolution was probably not driven by selection for high magnitude unilateral biting, and that increased masticatory muscle efficiency in humans is likely to be a secondary byproduct of selection for some function unrelated to forceful biting behaviors. These results are consistent with the hypothesis that a shift to softer foods and/or the innovation of pre-oral food processing techniques relaxed selective pressures maintaining craniofacial features favoring forceful biting and chewing behaviors, leading to the characteristically small and gracile faces of modern humans. 
1 Human feeding biomechanics: performance, variation, and 2 functional constraints

3

4

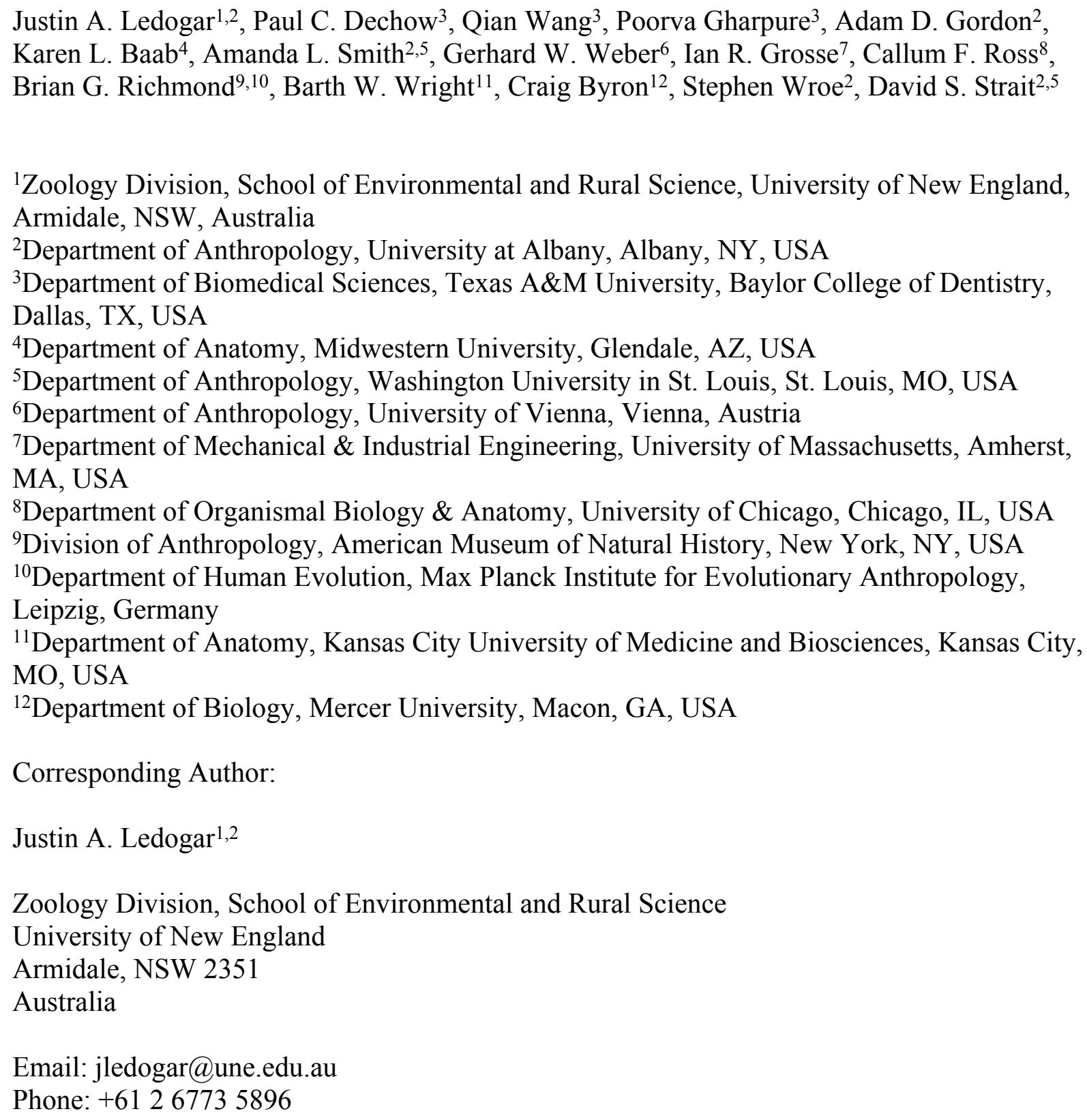


46

47

48

\section{ABSTRACT}

The evolution of the modern human (Homo sapiens) cranium is characterized by a reduction in the size of the feeding system, including reductions in the size of the facial skeleton, postcanine teeth, and the muscles involved in biting and chewing. The conventional view hypothesizes that gracilization of the human feeding system is related to a shift toward eating foods that were less mechanically challenging to consume and/or foods that were processed using tools before being ingested. This hypothesis predicts that human feeding systems should not be well-configured to produce forceful bites and that the cranium should be structurally weak. An alternate hypothesis states that the modern human face is adapted to generate and withstand high biting forces. We used finite element analysis (FEA) to test two opposing mechanical hypotheses: that compared to our closest living relative, chimpanzees (Pan troglodytes), the modern human craniofacial skeleton is 1) less well configured, or 2) better configured to generate and withstand high magnitude bite forces. We considered intraspecific variation in our examination of human feeding biomechanics by examining a sample of geographically diverse crania that differed notably in shape. We found that our biomechanical models of human crania had broadly similar mechanical behavior despite their shape variation and were, on average, less structurally stiff than the crania of chimpanzees during unilateral biting when loaded with physiologically-scaled muscle loads. Our results also show that modern humans are efficient producers of bite force, consistent with previous analyses. However, highly tensile reaction forces were generated at the working (biting) side jaw joint during unilateral molar bites in which the chewing muscles were recruited with bilateral symmetry. In life, such a configuration would have increased the risk of joint dislocation and constrained the maximum recruitment levels of the masticatory muscles on the balancing (non-biting) side of the head. Our 
69 results do not necessarily conflict with the hypothesis that anterior tooth (incisors, canines,

70 premolars) biting could have been selectively important in humans, although the reduced size of

71 the premolars in humans has been shown to increase the risk of tooth crown fracture. We

72 interpret our results to suggest that human craniofacial evolution was probably not driven by

73 selection for high magnitude unilateral biting, and that increased masticatory muscle efficiency

74 in humans is likely to be a secondary byproduct of selection for some function unrelated to

75 forceful biting behaviors. These results are consistent with the hypothesis that a shift to softer

76 foods and/or the innovation of pre-oral food processing techniques relaxed selective pressures

77 maintaining craniofacial features favoring forceful biting and chewing behaviors, leading to the

78 characteristically small and gracile faces of modern humans. 


\section{INTRODUCTION}

95

96

97

98

Human craniofacial architecture is extreme among living primate species. In particular, modern humans (Homo sapiens) exhibit a tall braincase and a small and short maxilla which distinguishes them from even our closest living relatives, the chimpanzees and bonobos of genus Pan (Fleagle, Gilbert \& Baden, 2010). Reductions in the size and prognathism of the face, combined with increases in neurocranial globularity, have also been shown to differentiate modern humans from some extinct members of the genus Homo (Lieberman, McBratney \& Krovitz, 2002). Homo exhibits an even more pronounced reduction in the size and robusticity of the facial skeleton, as well as in the size of the postcanine dentition and masticatory muscles (e.g., Robinson, 1954; Rak, 1983; Demes \& Creel, 1988), relative to australopiths, an extinct informal group of early hominins from which modern humans are likely to be descended (e.g., Walker, 1991; Wood, 1992; Skelton \& McHenry, 1992; Strait, Grine \& Moniz, 1997; Strait \& Grine, 2004; Kimbel, Rak \& Johanson, 2004; Berger et al., 2010). Theories purporting to explain the adaptive significance of masticatory reduction in Homo frequently stress the importance of changes in diet, usually involving a shift to foods that require less extensive intra-oral processing (e.g., Robinson, 1954; Rak, 1983; Brace, Smith \& Hunt, 1991; Wrangham et al., 1999; Lieberman et al., 2004; Ungar, Grine \& Teaford, 2006; Wood, 2009). However, Wroe et al. (2010) suggest that modern human crania are instead adapted to produce forceful bites, based on their conclusion that the human feeding apparatus is mechanically efficient, requires less muscle force than most other hominoids in order to generate comparable bite reaction forces, and should 
114 therefore require a less robust structure. This paper evaluates these two alternatives by

115 comparing feeding biomechanics in modern H. sapiens to that of chimpanzees (Pan troglodytes).

116 A conventional view of cranial gracilization in the lineage leading to modern Homo states

117 that this process was spurred by the development of stone tool technologies (e.g., Ungar, Grine

$118 \&$ Teaford, 2006), as tool use reduces food particle size (Lucas, 2004), allowing a reduced bite

119 force per chew and/or fewer chews per feeding bout (Lucas \& Luke, 1984; Agrawal et al., 1997;

120 Zink \& Lieberman, 2016). Under this hypothesis, tool use reduces the selective advantage

121 offered by anatomical features that increase muscle force leverage and/or buttress the face

122 against feeding loads. In addition to tool use, increased reliance on meat eating may have played

123 a role in the initial stages of masticatory reduction in early Homo (Lieberman, 2008; Ungar,

124 2012; Zink \& Lieberman, 2016). Further gracilization of the jaws and teeth is hypothesized to

125 have occurred with the advent of cooking, which may have been practiced by H. erectus

126 (Wrangham, 2009; Organ et al., 2011), by reducing masticatory stresses (Lieberman et al., 2004;

127 Lucas, 2004) and increasing digestive efficiency (Wrangham et al., 1999; Carmody \&

128 Wrangham, 2009; Carmody, Weintraub \& Wrangham, 2011; Groopman, Carmody \&

129 Wrangham, 2015). If gracilization in Homo is a consequence of the removal of selection pressure

130 to maintain and resist high magnitude or repetitive bite forces, then human feeding systems

131 should not be optimized to produce high biting forces and the cranium could be structurally weak

132 (i.e., exhibit high stress and strain when exposed to feeding loads).

133 The hypothesis described above is opposed by an alternative interpretation of human

134 feeding mechanics. A paradox of the human cranium is that the marked facial orthognathism

135 exhibited by recent modern humans increases the mechanical advantage (i.e., leverage) of the

136 muscles responsible for elevating the mandible, allowing humans to generate a given bite force 
137 with relatively low muscular effort (Spencer \& Demes, 1993; O’Connor, Franciscus \& Holton,

138 2005; Lieberman, 2008, 2011; Wroe et al., 2010; Eng et al., 2013). Many studies interpret bite

139 force efficiency among primate species as being significant in an adaptive sense (Rak, 1983;

140 Strait et al., 2013; Smith et al., 2015a; Ross \& Iriarte-Diaz, 2014), with increases in leverage

141 predicted for species that rely on foods that require forceful biting in order to be processed (e.g.,

142 hard seeds or nuts). Therefore, high biting leverage among humans seemingly contrasts with the

143 hypothesis that the human craniofacial skeleton has experienced relaxed selection for traits that

144 favor forceful biting and chewing behaviors (e.g., Brace, Smith \& Hunt, 1991; Lieberman et al.,

145 2004; Ungar, Grine \& Teaford, 2006; Wood, 2009). However, Wroe et al. (2010) present an

146 alternative view based on their analysis of modern human, extant ape, and fossil australopith

147 feeding biomechanics. Using finite element analysis (FEA), Wroe et al. (2010) found that their

148 human model was mechanically more efficient at producing bite forces than the other hominoids

149 in their sample. Additionally, they found that the human cranium experienced stresses similar to

150 those in 3 of the 5 other species when models were scaled to the same surface area and bite force,

151 including Pan. Consequently, Wroe et al. (2010) conclude that the human skull need not be as

152 robust in order to generate, or sustain, bite reaction forces comparable to those of other

153 hominoids, and that powerful biting behaviors may have been selectively important in shaping

154 the modern human cranium.

155 Here, we use FEA to test two opposing mechanical hypotheses: that relative to

156 chimpanzees, the modern human craniofacial skeleton is 1) less well configured, or 2) better

157 configured to generate and withstand high magnitude unilateral bite forces. Our analysis builds

158 on previous research into human craniofacial function (e.g., Lieberman, 2008; Wroe et al., 2010;

159 Szwedowski, Fialkov \& Whyne, 2011; Maloul et al., 2012) by examining masticatory 
160 biomechanics within the context of the constrained lever model (Greaves, 1978; Spencer and

161 Demes, 1993; Spencer, 1998, 1999), which predicts that bite force production in mammals is

162 constrained by the risk of generating distractive (tensile) forces at the working (biting) side TMJ.

163 Under this model, during unilateral biting, reaction forces are produced at the bite point and the

164 working and balancing (non-biting) side TMJs. These three points form a "triangle of support",

165 and the line of action of the resultant vector of the jaw elevator muscle forces must intersect this

166 triangle in order to produce a "stable" bite in which compressive reaction forces are generated at

167 all three points (Fig. 1A). The resultant vector lies in the midsagittal plane when the muscles are

168 recruited with bilateral symmetry and will pass through the triangle of support during bites on

169 the incisors, canines, and premolars. However, molar biting changes the shape of the triangle

170 such that a midline muscle result may lie outside of the triangle of support. If this occurs, a

171 distractive (tensile) force is generated in the working side TMJ that "pulls" the mandibular

172 condyle from the articular eminence (Fig. 1B). In the case of the mammalian jaw, the soft tissues

173 of the TMJ are well suited to resist compressive joint reaction forces in which the mandibular

174 condyle is being "driven" into the cranium, but they are poorly configured to resist distractive

175 joint forces in which the condyle is being "pulled away" from the cranium (Greaves, 1978).

176 Mammals, including humans (Spencer, 1998), avoid this by reducing the activity of the chewing

177 muscles on the balancing side during bites on the posterior teeth. This draws the muscle resultant

178 vector toward the working side and back within the triangle, but the total muscle force available

179 for biting is reduced, thereby reducing peak bite force magnitudes. Thus, although one might

180 expect that a bite on a distal tooth would produce an elevated bite force due to a short load arm

181 (per a given muscle force), this effect is mitigated by the constraint that the muscle force vector

182 must lie within the triangle of support. A finding that constraints on bite force production were 
183 especially strong in humans would be consistent with the hypothesis that the human cranium is

184 poorly configured to generate high unilateral bite forces, and inconsistent with the opposing

185 hypothesis.

186 We further build on previous work by considering intraspecific variation in our analysis

187 of human feeding biomechanics. Our prior work has shown that high degrees of intraspecific

188 variation in cranial shape need not necessarily produce a high degree of intraspecific mechanical

189 variation (Smith et al., 2015b), implying that mechanical patterns are conservative and reflect an

190 underlying common geometry that may be overlain by skeletal traits that can vary without

191 dramatically altering the fundamental mechanical framework of the cranium. A caveat, however,

192 is that Smith et al. (2015b) examined only one species, P. troglodytes. Thus, it has yet to be

193 established if this pattern is generalizable across primates (or other vertebrates). Accordingly, we

194 examined mechanical variation among a sample of geographically diverse human crania found to 195 differ notably in shape.

196

197 MATERIALS \& METHODS

198 Analysis of human cranial shape variation and selection of specimens for FEA

We analyzed finite element models (FEMs) of six crania lying at the extremes of human variation, as well as one "average" specimen found to conform closely to an average shape. To select specimens, we analyzed shape variation within a sample of modern human (H. sapiens) crania using previously collected geometric morphometric (GM) data (Baab et al., 2010). We analyzed 85 landmarks collected from a sample of 88 Holocene human crania housed at the American Museum of Natural History (AMNH) (Tables 1, 2). These included mainly facial 
206 on facial biomechanics in this study. This sample includes individuals from diverse regions

207 across the globe, and provides a cross-section of populations that differ in cranial robusticity

208 (Baab et al., 2010). Landmark data from these 88 specimens were converted to shape coordinates

209 by Generalized Procrustes analysis (e.g., Bookstein, 1991; Slice, 2005) and analyzed using

210 principal components analysis (PCA). We found that the first 3 principal components (PCs)

211 described 39\% of the shape variation in our sample (Fig. 2). In order to maximize shape-related

212 biomechanical variation in our FEMs, we considered variation from all 88 PCs when selecting

213 specimens to be modeled. We first determined those individuals exhibiting the largest distances

214 from the group centroid (i.e., consensus shape), calculated as Euclidean distance using all 88 PCs

215 (Table 3). From among these individuals, we chose the six specimens that exhibited the largest

216 pairwise distances, excluding insufficiently preserved crania, those missing many teeth, and

217 those unavailable for loan (Table 4). These six "extreme" modern human crania included: one

218 male and one female Khoe-San from South Africa (AMNH VL/2463 and AMNH VL/2470,

219 hereafter referred to as "KSAN1" and "KSAN2"); a male from Greifenberg, Austria (AMNH

220 VL/3878, “BERG”); a female from the Malay Archipelago (AMNH 99/7889, "MALP”); a male

221 from the Tigara culture at Point Hope, Alaska (AMNH 99.1/511, "TIGA”); and a male from

222 Ashanti, West Africa (AMNH VL/1602, "WAFR"). An additional specimen, a Native American

223 male from Grand Gulch, Utah (AMNH 99/7365, "GRGL”), was chosen as an "average"

224 representative of human cranial shape based on its close proximity (i.e., small Euclidean

225 distance) to the group centroid and its availability for loan (see Table 3). Note that this individual

226 was incorrectly transcribed as AMNH 99/7333 by Ledogar (2015).

228 Creation of finite element models from "extreme" and "average" human specimens 


\section{Construction of solid models}

The seven specimens chosen for analysis were CT-scanned at Penn State's Center for Quantitative Imaging (pixel size $=0.16 \mathrm{~mm}$ ) and the 2D digital image stacks were used to create seven solid meshes (Fig. 3) using Mimics v 14.0 (Materialise, Ann Arbor, MI, USA), following the methods outlined by Smith et al., 2015 (a,b). Mandibles corresponding to the seven crania (except for BERG and KSAN2, which lacked mandibles; see below) were also scanned so that they could be used to direct muscle force vectors in the loading simulations described below. The crania were solid-meshed at similar densities using tet 4 elements (element count:

$\mathrm{GRGL}=2,118,350 ;$ BERG=1,928,931; KSAN1=1,620,112; KSAN2=1,392,417;

MALP=1,323,093; TIGA=2,059,433; WAFR=1,831,053). Solid meshes were then imported as Nastran (NAS) files into Strand7 (Strand7 Pty Ltd) FEA software.

We created two sets of human FEMs that differed in their assigned muscle force and bone properties. One set of human FEMs (“ALL-HUM" models) was assigned human properties for bone tissue and masticatory muscle force, whereas chimpanzee properties were applied to the second set ("CHIMPED" models). The ALL-HUM models provide the most realistic assessment of human cranial mechanics, in terms of the predicted strains and bite forces. These models also allow for a more thorough examination of intraspecific variation in humans. In contrast, the CHIMPED models permit direct comparisons between our humans FEMs and our previously analyzed FEMs of chimpanzees and fossil hominins (Smith et al., 2015a,b). These comparisons focus on shape-related differences in mechanical performance that are free of the effects of differences in cranial size and bone material properties. Therefore, the comparisons between the CHIMPED human models and the chimpanzee data from Smith et al. $(2015 \mathrm{a}, \mathrm{b})$ most directly 
251 address our mechanical hypothesis described above because the hypotheses relate specifically to

252 the mechanical consequences of shape differences.

253

254 Material properties of tissues

255 Human cortical bone material properties assigned to the ALL-HUM models were

256 collected from various locations across the craniofacial skeletons of two fresh-frozen human

257 cadavers (female, aged 22; male, aged 42) by measuring their resistance to ultrasonic wave

258 propagation (see Supplementary Information). Previous studies show that freezing has only a

259 very minimal effect on ultrasonic measurements and elasticity of cortical bone (Zioupos et al.,

260 2000). For each location sampled, the elastic (Young's) modulus in the axis of maximum

261 stiffness (E3) was averaged between the human donors and used to distribute spatially

262 heterogeneous isotropic material properties throughout the seven human FEMs using a method

263 (Davis et al., 2011) analogous to the diffusion of heat through a highly conductive material. To

264 achieve this, values at each of the sampled locations, which ranged from $17.92 \mathrm{GPa}$ to $25.52 \mathrm{GPa}$

265 (mean=20.61 GPa, SD=1.92), were converted to temperatures and distributed throughout the

266 cortical volume of the FEM. The elastic modulus of cortical bone was then set to vary with

267 temperature during the subsequent loading analysis, with any thermally-induced strains removed

268 from the analysis. For Poisson's ratios, models were each assigned the average of the sampled

269 locations $(v 23=0.293)$. The same procedure was used to diffuse chimpanzee material properties

270 to the CHIMPED model variants using data collected from a cadaveric female chimpanzee at 14

271 craniofacial regions (Smith et al., 2015a,b). In both the ALL-HUM and CHIMPED sets of model

272 variants, homogeneous isotropic properties were used to model both trabecular bone $(E 3=637$

$273 \mathrm{MPa} ; v 23=0.28)$ and enamel $(E 3=80,000 \mathrm{MPa} ; v 23=0.28)$, following Smith et al. $(2015 \mathrm{a}, \mathrm{b})$. 
Muscle forces and constraints

Jaw adductor muscle forces were applied to both sets of FEMs for the anterior

277

278

279

280

281

282

283

284

285

286

287

288

289

290

291

292

293

294

295

296 temporalis, superficial masseter, deep masseter, and medial pterygoid under the assumption that

the chewing muscles were acting at peak activity levels on both sides of the cranium. These

loads allow an estimate of the maximum bite force produced by each individual. In the ALL-

HUM variants, muscle forces were applied based on muscle physiological cross-sectional area

(PCSA) data reported by van Eijden, Korfagen \& Brugman (1997), with forces corrected to

account for pennation and differences in gape during fixation using formulae from Taylor \&

Vinyard (2013). Corrected PCSAs were then used to calculate forces in Newtons (N) such that

each $\mathrm{cm}^{2}$ of muscle was equivalent to $30 \mathrm{~N}$ (Murphy, 1998). These unscaled forces were applied

to the "average" specimen (GRGL), while the six "extreme" variants were applied forces that

were either scaled up or down based on differences in model size (Table 5), with size represented

by model volume (i.e., the summed volume of all tet4 elements in $\mathrm{mm}^{3}$ ) to the two-thirds power.

This muscle force scaling procedure removes the effects of differences in model size on stress,

strain, and strain energy density from the mechanical results (Dumont, Grosse \& Slater, 2009;

Strait et al., 2010). The CHIMPED model variants were also assigned forces that were scaled

dependent on their size using PCSA data from an adult female chimpanzee (Strait et al., 2009;

Smith et al., 2015a,b). However, rather than scaling the FEMs around the "average" specimen

(GRGL), we scaled the forces applied to the CHIMPED models (see Table 5) from the baseline

chimpanzee model used for scaling purposes (PC1+) in the analysis by Smith et al. (2015b),

permitting size-free comparisons between humans and chimps. For both sets of muscle loadings,

plate elements modeled as 3D membrane were "zipped" at their nodes to the surface faces of tet4 
297 elements representing each muscle's origin. The scaled muscle forces for each set of analyses

298 were applied using Boneload (Grosse et al., 2007) to the normal surfaces of the plate elements as

299 tractions directed toward their respective insertions on the mandible, with the mandible slightly

300 depressed and the condyles translated onto the articular eminences (Dumont, Piccirillo \& Grosse,

301 2010). Mandibles were only used here to direct these vectors. In the case of the BERG specimen,

302 which was lacking its mandible, a scaled version of the GRGL mandible was used to define the

303 orientation of muscle force vectors. Similarly, a scaled version of the KSAN1 mandible was used

304 to replace the missing mandible in KSAN2.

305 For both sets of biting simulations, each of the seven FEMs was oriented such that one of

306 three axes (i.e., X, Y, or Z) was parallel to the occlusal plane. Each model was constrained at a

307 single node against translation in all axes at the working-side TMJ, while the balancing-side TMJ

308 was constrained only in the superoinferior and anteroposterior directions (Strait et al., 2009;

309 Smith et al., 2015a,b), thus creating an axis of rotation around the TMJs. Models were subjected

310 to simulations of left premolar $\left(\mathrm{P}^{3}\right)$ and left molar $\left(\mathrm{M}^{2}\right)$ biting by constraining a node in the

311 center of occlusal surface in each tooth, respectively, in the superoinferior direction. These

312 constraints generated strains in the craniofacial skeleton, as well as reaction forces at the TMJs

313 and bite point, upon the application of muscle forces.

Analysis of model output parameters

316 Following Smith et al (2015a,b), we displayed global strain patterns using strain maps.

317 These maps are analogous to histograms in that they illustrate strain magnitudes at thousands of

318 nodes simultaneously, but have the added advantage of preserving spatial information. In

319 addition, we collected strain data generated by each FEM from surface elements at 14 locations 
320 across the craniofacial skeleton (Fig. 4). These locations correspond to those included in

321 previous in vitro and in silico (e.g., FEA) studies on primate feeding biomechanics (e.g.,

322 Hylander and Johnson, 1991, 1997; Ross et al., 2011; Smith et al., 2015a,b). At each location, we

323 examined several strain metrics from each of the seven FEMs in order to understand patterns of

324 deformation. These included maximum principal strain (tension), minimum principal strain

325 (compression), maximum shear strain (maximum principal strain - minimum principal strain),

326 von Mises strain (distortional strain or non-isometric strain), and strain energy density (SED, the

327 strain energy stored at a given point). Additionally, strain mode, the absolute value of maximum

328 principal strain divided by minimum principal strain, was recorded for each location. This

329 measure indicates whether tension or compression is dominant at a given location.

330 Data on the reaction forces generated at constrained nodes (i.e., the bite point and two

331 TMJs) were recorded in Newtons $(\mathrm{N})$. Reaction forces at the $\mathrm{P}^{3}$ and $\mathrm{M}^{2}$ were recorded relative to

332 the occlusal plane, while reaction forces at the left and right TMJs were recorded and compared

333 relative to a user-defined "triangle of support" Cartesian coordinate system, with one of three

334 axes perpendicular to a reference plane defined by the "triangle of support" formed by the

335 constrained nodes at the bite point and two articular eminences (Smith et al., 2015a,b). The

336 efficiency of bite force production at a given bite point in each model was also compared using

337 the mechanical advantage (MA), a measure of masticatory muscle efficiency or leverage,

338 calculated as the ratio of bite force output to muscle force input.

339 In the evaluation of our mechanical hypothesis, we first inspected data collected from the

340 ALL-HUM models for large levels of intraspecific variation that could potentially invalidate the

341 functional significance of our results. Strain magnitudes and SED at each of the 14 sampled

342 locations were examined for large differences between individuals, in addition to a comparison 
343 of coefficients of variation (CVs) at specific locations. Differences in the spatial patterning of

344 strain magnitudes between the ALL-HUM models were also compared using strain maps, in

345 addition to variation in biting efficiency (i.e., MA). Lastly, we also calculated CVs for von Mises

346 strain and MA in the CHIMPED model variants for direct comparison with the chimpanzee CVs

347 reported by Smith et al. (2015b) using the Fligner-Killeen test for equal CVs.

348 To analyze relative mechanical performance in our human FEMs, we focused on

349 comparisons between the CHIMPED humans and our previously analyzed FEMs of chimpanzee

350 crania (Smith et al., 2015b). Specifically, we compared the magnitudes of von Mises strain,

351 considered to be a key metric in assessing regional bone strength (Keyak \& Rossi, 2000), at the

35214 sampled locations, as well as differences in biting efficiency, between humans and chimps.

353 We tested for significant differences between species using the Mann-Whitney $U$ test.

\section{In vitro validation of specimen-specific human cranial FEM}

Data on in vitro bone strain collected during simulated $\mathrm{P}^{3}$ biting in a cadaveric human

357 head were used to validate our results. As noted above, two human heads were used to gather 358 data on the properties of craniofacial cortical bone. Before the removal of bone samples, the male specimen was CT-scanned, and strain data from 14 craniofacial locations were collected

360 during a series of in vitro loading analyses (see Supplementary Information). Digital images of

361 the specimen were then used to construct an eighth FEM, the in vitro loadings were replicated

362 using FEA, and strain data were collected from the FEM at locations corresponding to the 14

363 gage sites. The in vitro and in silico strain data were then compared in order to establish the

364 degree to which assumptions regarding geometry and material properties introduce error into an

365 FEM, where error is represented by the differences between the in vitro (observed) and in silico 
366 (expected) results, divided by the expected results. These data were also analyzed using ordinary

367 least squares (OLS) regression. Lastly, the orientations for both maximum and minimum

368 principal strain in FEM were visually compared to those recorded during the in vitro loadings.

369

370

371

372

373

374

375

376

377

378

379

380

381

382

383

384

385

386

387

388

\section{RESULTS}

\section{In vitro validation of specimen-specific human cranial FEM}

Strain magnitudes recorded during in vitro $\mathrm{P}^{3}$ loadings of the human cadaveric specimen and the results of the specimen-specific FEA are listed in Table 6. Comparisons of these data reveal that the specimen-specific FEM generated strains very similar in magnitude to those generated during the in vitro loadings. Results of the regression analysis on log-transformed strain data confirm a close correspondence between in vitro and in silico results, with significant regressions of $0.845 x+0.194\left(r^{2}=0.909, p<0.001\right)$ and $0.849 x+0.186\left(r^{2}=0.953, p<0.001\right)$ for maximum principal strain and minimum principal strain, respectively. However, assumptions regarding geometry and material properties did introduce error into the FEM (see Table 6).

Visual inspection of principal strain orientations in the specimen-specific FEA reveals that orientations for both maximum principal strain and minimum principal strain at the 14 sampled locations were also very similar to those recorded from the 14 gage locations during the in vitro analysis (Fig. S3 - Fig. S7).

\section{Shape-related variation in human feeding biomechanics}

\section{Variation in strain magnitude and spatial patterning}

Box-plots of strain and SED distributions recorded from the ALL-HUM models at the 14 sampled locations during premolar $\left(\mathrm{P}^{3}\right)$ and molar $\left(\mathrm{M}^{2}\right)$ biting are shown in Fig. 5 (see also 
389 Tables S1 and S2). Despite notable differences in craniofacial morphology between the models, 390 comparisons of strain magnitudes reveal strong similarities. For $\mathrm{P}^{3}$ biting, the highest strain 391 magnitudes were experienced at the working nasal margin (Location 12), although on average 392 higher tensile strain magnitudes were generated at the working and balancing postorbital bars 393 (Locations 4 and 5). During $\mathrm{M}^{2}$ biting, the working zygomatic root (Location 8) was subjected to 394 the highest strain magnitudes, except that tension was greatest at the balancing postorbital bar. 395 During both bites, low strain magnitudes were generated along the supraorbital torus (Locations 396 397 1-3), the balancing zygomatic root (Location 9), balancing infraorbital (Location 11), and the zygomatic bodies (Locations 13 and 14). All FEMs of human crania were found to exhibit this general pattern.

Some regions of the face did exhibit large differences among individuals. In particular, the FEMs were found to differ in von Mises strain magnitude by as much as $210 \%$ at the nasal margin, which also has the highest $\mathrm{CVs}$ for all forms of strain during both $\mathrm{P}^{3}$ and $\mathrm{M}^{2}$ biting 402 (Table 7), with the exception of minimum principal strain at the working dorsal orbital (Location 2) and balancing infraorbital (Location 11) during $\mathrm{P}^{3}$ biting, SED at the working dorsal orbital (Location 2) during $\mathrm{P}^{3}$ biting, and the balancing zygomatic body (Location 14) for both bites. ALL-HUM models (Fig. 6), with a few exceptions. During premolar biting, only 3 locations varied with respect to strain mode (Locations 1, 10, 11), with only one FEM differing from the other models in each case. These three locations also differed in strain mode during molar biting, with Locations 1 and 10 exhibiting slightly higher levels of variation, in addition to variation in strain mode at Location 4. 
412 shape-related variation in von Mises strain magnitude and lower CVs than chimpanzees at the 14

413 sampled locations (Table 8). However, results of the Fligner-Killeen tests reveal that only 3 of

414 the 14 "gage sites" exhibit significant differences in CV values. Specifically, humans were found 415 to exhibit a significantly lower $\mathrm{CV}$ at the zygomatic arches during both $\mathrm{P}^{3}$ and $\mathrm{M}^{2}$ biting at the 416 working infraorbital during $\mathrm{P}^{3}$ biting.

\section{Variation in the spatial patterning of strain concentrations}

Despite some large differences in strain magnitude, the spatial patterning of strain

tensile and compressive (hence highly shearing) strains surrounding the root of the nasal margin,

frontal bending of the zygomae under the inferiorly directed pulling action of the masticatory

muscles, which generates tension at the zygomatic body and near the zygomaticomaxillary

427 junction, particularly at the working-side, and deforms the orbit such that it is tensed along an

428 inferolaterally-oriented axis and compressed along a superolaterally-oriented axis.

the ALL-HUM models (Fig. 8). As expected, all models exhibited lower strain magnitudes in the lower maxillary region during molar biting compared to premolar biting, but higher concentrations of compressive strain at the working zygomatic root. Molar biting was also associated with the same type of frontal bending, zygomatic torsion, and orbital deformation that 
434 was observed for premolar biting, with relatively large concentrations of strain at the postorbital 435 bars, orbital margins, and medial infraorbital.

In their study of chimpanzee biomechanical variation, Smith et al. (2015b) compared

437

438

439

440

441

442

443

444

445

446

447

448

449

450

451

452

453

454

455

456

color maps of principal strain magnitudes in their 6 models with the scales normalized to an average of 10 landmarks (Locations 1-5, 8-12). They suggest that, by illuminating similarities and differences between individuals in the concentrations of relatively high and low strain concentrations through this normalization step, such "relative strain" maps strain may be particularly informative in comparative analyses of craniofacial function. When viewed in this manner (Fig. 9), the CHIMPED human models more clearly reveal a shared pattern of facial deformation that differs from that of chimpanzees under identical loading conditions, which was predominantly characterized by torsion of the zygoma and resulting orbital deformation under the inferiorly-directed masseteric muscle force.

\section{Variation in bite force production and efficiency}

The ALL-HUM models exhibit moderate differences in bite force production and efficiency (mechanical advantage, MA) at $\mathrm{P}^{3}$ and $\mathrm{M}^{2}$ bite points (Table 9). With respect to bite force production, humans generated premolar bite forces that ranged from 333 to $507 \mathrm{~N}$ when loaded with scaled masticatory muscle forces. The MA range for premolar biting was 0.34-0.43 with all but one individual (WAFR) occupying a narrower range of 0.39-0.43. Molar bite forces ranged from 496 to $756 \mathrm{~N}$. In terms of leverage, most FEMs exhibited molar MAs of 0.57-0.64, but with the WAFR model again being considerably less efficient (0.53).

When compared to the chimpanzee data in Smith et al. (2015a), the CHIMPED human models analyzed here were found to exhibit somewhat lower ranges of variation in biting MA. 
457 However, results of the Fligner-Killeen tests reveal no significant differences in CV values

458 between the species at either the $\mathrm{P}^{3}(\operatorname{chimp}=8.67$, human=5.65; $\mathrm{p}=0.18)$ or $\mathrm{M}^{2}(\mathrm{chimp}=8.11$,

459 human $=6.67 ; \mathrm{p}=0.13$ ) bite point.

460

461

Variation in reaction forces generated at the temporomandibular joints

462

During premolar biting, all seven of the ALL-HUM models generated strongly

463

compressive reaction forces at both TMJs (see Table 9), similar to the results for chimpanzees

464

(Smith et al., 2015b). However, unlike in chimpanzees, $\mathrm{M}^{2}$ biting generated distractive (tensile)

465

reaction forces at the working-side TMJ that would have "pulled" the mandibular condyle away

from the articular eminence in five of the seven models. In order to remove distractive forces,

467

these models required reductions in the muscle force applied to the balancing-side, which ranged

from $5 \%$ to $15 \%$ (see Table 9 ). Interestingly, when loaded with chimpanzee muscle forces, all

469

seven of the CHIMPED human models exhibit distractive forces in the working TMJ during $\mathrm{M}^{2}$

470

biting, with larger muscle force reductions required to eliminate the distraction (see below).

471

472

Biomechanical "performance" of human feeding

Structural stiffness of the human craniofacial skeleton

Direct comparisons of shape-related mechanical performance between our human FEMs and our previously analyzed chimpanzee FEMs (Smith et al., 2015a,b) were permitted by the CHIMPED models. These comparisons reveal that the human craniofacial skeleton is less stiff and experiences von Mises strains that are elevated relative to those experienced by chimpanzees when subjected to identical loading conditions (Fig. 10). Several of the sampled locations were

479 found to experience significantly higher magnitudes in humans during both $\mathrm{P}^{3}$ and $\mathrm{M}^{2}$ biting 
480 following the results of Holm-Bonferroni-corrected Mann-Whitney $U$ tests (Table 10). These

481 included the working nasal margin (Location 12), postorbital bars (Locations 4 and 5), working

482 zygomatic root (Location 8), and the working dorsal orbital (Location 2). However, strains at the

483 mid-zygomatic arches in humans were within the range observed for chimpanzees (which are

484 extremely variable). Additionally, human zygomatic bodies were found to be structurally stiff,

485 with significantly lower von Mises strain magnitudes than chimpanzees.

486

Human bite force production and mechanical efficiency

488

489

490

491

492

493

494

495

496

497

498

499

500

501

502
Analysis of our CHIMPED human FEMs reveals that human crania are capable of generating bite forces with higher mechanical efficiency than chimpanzees (Fig. 11). Pairwise comparisons using the Mann-Whitey $U$ test demonstrate that these differences are significant at both $\mathrm{P}^{3}(U=1.5, \mathrm{z}=-2.73$, exact $\mathrm{p}=0.003)$ and $\mathrm{M}^{2}(U=1, \mathrm{z}=-2.79$, exact $\mathrm{p}=0.002)$ bite points. However, unlike chimpanzees, all seven of the CHIMPED human models generated highly distractive (tensile) reaction forces at the working-side TMJ during molar biting. Therefore, molar biting in humans increases the risk of having the muscle resultant vector fall outside the triangle of support. To bring the joint back into compression, a reduction in balancing side muscle force of 15\%-30\% was required (Table 11).

\section{DISCUSSION}

\section{In vitro validation}

In order to validate the findings of our mechanical analysis, we compared in vitro bone strain in a cadaveric human head during simulated $\mathrm{P}^{3}$ biting to the results of a specimen-specific FEA. We found the results of our specimen-specific FEA corresponded quite well with in vitro 
503 data. In addition to the notable similarities in strain orientation at the 14 sampled locations,

504 results of the regression analysis reveal that FEA can predict in vitro strain magnitudes with a

505 high degree of accuracy $\left(r^{2}\right.$ values $\left.>0.9\right)$. Similarly, Nagasao et al. (2005) were able to validate a

506 dry bone human cranium with a high degree of accuracy $\left(r^{2}=0.989\right)$. However, these authors

507 examined only 2 gage sites and they simulated biting by applying forces to teeth, thus omitting

508 the impact of muscle loading. A greater number of sites were included in an analysis by

509 Szwedowski, Fialkov \& Whyne (2011), who found that their FEM results predicted in vitro data

510 with an $\mathrm{r}^{2}$ of 0.73 . Toro-Ibacache et al. (2015) also applied point loads to a cadaveric human

511 head and validated strains at two locations in a specimen-specific FEM, finding broad

512 similarities.

513 Although we found excellent correspondence between in vitro and in silico results, it is

514 clear that FEA does incorporate error (see Table 6). This error was deceptively large at some

515 "gage sites," particularly in areas of low strain. For example, error for maximum principal strains

516 at the balancing dorsal orbital (Location 3) was $80 \%$, but this represents a difference between

517 experimental and FEA results of only 2.67 microstrain $(\mu \varepsilon)$. Generally speaking, this is not a

518 meaningful difference in the context of vertebrate feeding biomechanics, where some regions of

519 the cranium can experience strain in the thousands of microstrain. However, some moderately

520 strained areas exhibited high error percentages. In particular, the working infraorbital validated

521 well for minimum principal strain, but error for maximum principal strain was nearly $50 \%$. This

522 discrepancy may be related to the morphology of the bone that forms the thin anterior wall of the

523 maxillary sinus, which is susceptible to large modeling errors (Maloul, Fialkov \& Whyne, 2011),

524 or could be a result of simplifications to the thin bones of the nasal cavity (see Toro-Ibacache et

525 al., 2015). 


\section{Mechanical variation}

We found that the ALL-HUM models exhibited generally low levels of shape-related

529

530

531

532

533

534

535

536

537

538

539

540

541

542

545

546

547

548

543 Craniofacial strength: Is the human face weak?

544 Our results suggest that the modern human craniofacial skeleton is structurally less

mechanical variation in strain magnitude and bite force production. Additionally, though some regions (e.g., the nasal margin) were found to exhibit large differences in strain magnitude, our human FEMs shared a common pattern of the spatial distribution of relatively high and low strain concentrations. These findings are similar to those of Smith et al. (2015b), who found broad similarities in strain patterning among on a sample of chimpanzee FEMs that differed notably in shape. Similarly, Toro-Ibacache, Zapata Muñoz \& O’Higgins (2015) found broad similarities between two notably distinct human cranial FEMs. Our finding that the ALL-HUM models exhibit low levels of mechanical variation supports the functional significance of the comparisons of shape-related mechanical performance made between our CHIMPED human FEMs and our previously analyzed chimpanzee FEMs (Smith et al., 2015a,b), which focused purely on mechanical differences resulting from geometrical/architectural variation in the craniofacial skeleton.

542 Mechanical performance in humans and chimpanzee strong, in terms of resistance to masticatory stress, than that of chimpanzees when subjected to identical loading conditions (i.e., same properties and constraints, muscle forces scaled to model size). In the CHIMPED variants of our human FEMs, most of the locations analyzed experienced von Mises strain magnitudes that were elevated relative to chimpanzees, in particular the 
549 working nasal margin, the postorbital bars, the working zygomatic root, and the working dorsal

550 orbital region. Exceptions to this pattern include the zygomatic arches, where strains were

551 bracketed by the range of values seen in chimp FEMs, and the prominence of the zygomatic

552 body (i.e., the "cheek bone"), which is apparently strong in modern humans.

553 During unilateral $\mathrm{P}^{3}$ biting, the nasal margin of modern humans experienced von Mises

554 strains that were on average more than $350 \%$ greater than chimpanzees. Similarly, previous

555 investigations identify the "root" of the nasal margin to be an area of high stress and strain

556 during masticatory loading in humans (Endo, 1965, 1966; Arbel, Hershkovitz \& Gross, 2000;

557 Szwedowski, Fialkov \& Whyne, 2011; Maloul et al., 2012). This region is often described as a

558 pillar-like structure (Benninghoff, 1925; Bluntschili, 1926), or section of a frame-like structure

559 (Görke, 1902; Endo, 1965, 1966), that resists mainly compression during anterior tooth biting.

560 The results of our analysis are in general agreement with these findings, except that tension at the

561 nasal margin was also found to be high in magnitude, indicating intense bending and shearing of

562 the lower maxillary region during anterior tooth biting (see Fig. 7 and Fig. 9).

563 In addition to the nasal margin, the postorbital bars of the human FEMs were also found

564 to experience highly elevated von Mises strain magnitudes compared to chimpanzees. However,

565 adjacent regions, including the zygoma/zygomatic body ("cheek bone") region and zygomatic

566 arch, were found to be similar in strength to the lower end of the chimpanzee range. Mechanical

567 analyses of Paranthropus boisei and Australopithecus africanus (Smith et al., 2015a) show a

568 similar pattern of relatively low strains in the zygomatic body. Smith et al. (2015a) suggest that

569 the structural strength of the zygomatic body in australopiths could be adaptively significant,

570 offering as one possibility that it serves to reduce strains in the nearby zygomatico-maxillary

571 suture. In pigs, it has been demonstrated that unfused sutures can fail at relatively modest stress 
572 levels (e.g., Popowics \& Herring, 2007), so some bony facial regions may serve to shield nearby

573 sutures from masticatory stresses rather than bone itself (Wang et al., 2012). Among smaller-

574 faced modern human crania, the zygomatico-maxillary suture may be especially prone to

575 experiencing relatively large masticatory stresses. In our FEMs, the largest strains in this region

576 of the mid-face were generated medial to the zygomatico-maxillary suture. The location of these

577 elevated strain magnitudes corresponds roughly to the location of facial fractures experienced

578 commonly during physical altercations (Ellis, El-Attar \& Moos, 1985). Facial fractures are also

579 common at the postorbital bar, as opposed to the zygomatic body or zygomatico-maxillary

580 suture, when the zygomatic body is exposed to traumatic blows (Ellis, 2012; Pollock, 2012).

581 Therefore, it is possible that the strength of the human zygomatic body, and perhaps the relative

582 weakness of the postorbital bar, is related to diverting stress from sutures that might otherwise

583 fail under relatively lower stress magnitudes.

584 In addition to the zygomatic body ("cheek bone") region, humans were found to exhibit

585 lower average von Mises strains and markedly lower peak strains than chimpanzees at the mid-

586 zygomatic arch, although human values were bracketed by the range of chimp values. This

587 potentially reflects differences in arch length. Specifically, the size of the temporalis muscle,

588 which is correlated with the area of the infratemporal fossa (Weijs \& Hillen, 1984), is

589 significantly reduced in humans compared to that of chimpanzees (Taylor \& Vinyard, 2013).

590 Demes \& Creel (1988) show that the area of the infratemporal fossa is nearly half that of

591 chimpanzees, meaning that the total length of the zygomatic arch is also reduced. Bone strain

592 analyses demonstrate that the arch is subjected to sagittal bending, as well as torsion along its

593 long axis (e.g., Hylander, Johnson \& Picq, 1991; Hylander and Johnson, 1997; Ross, 2001; Ross

594 et al., 2011). Predictions based on beam theory therefore suggest that a decrease in the length of 
595 the arch will lessen these bending and torsional moments, whereas a reduction in the height

596 and/or breadth of the arch will weaken it under bending and shear, respectively.

597 Functional interpretations based on the morphology of the zygomatic arch are

598 complicated by the fact that the temporalis fascia has been hypothesized to stabilize it from the

599 inferiorly-directed pulling action of the masseter muscle (Eisenberg \& Brodie, 1965). Curtis et

600 al. (2011) tested this hypothesis using FEA and found that models that do not include the

601 temporalis fascia will overestimate strains in the arch and surrounding regions, including the

602 postorbital bar and infraorbital. However, they also found that their models lacking a fascia

603 generated strains more similar in magnitude to those collected during in vivo experiments

604 (Hylander, Johnson \& Picq, 1991; Hylander and Johnson, 1997; Ross, 2001; Ross et al., 2011).

605 Similarly, previous FEA studies on primate crania that have not included a modeled fascia (e.g.,

606 Ross et al., 2005, 2011; Strait et al., 2005) also find broad agreement with in vivo data.

607 Therefore, we did not feel that it was necessary to include this structure in our FEMs.

608 Importantly, Curtis et al. (2011) did not actually model the temporalis fascia, rather, they applied

609 external forces along the margin of the attachment of the fascia. This procedure assumes that the

610 load transferred to bone by the fascia is evenly distributed around its perimeter. However, the

611 fascia is subjected to load by the inferiorly directed force produced by those temporalis fibers

612 that arise off of the deep surface of the fascia. This force should elevate tension in the fascia

613 along its superior margin (i.e., where it arises off of the superior temporal line) while reducing

614 tension along its inferior margin (i.e., along the arch). This factor may mitigate the role of the

615 fascia in resisting the contraction of the masseter muscle.

616 Although the brow ridges are not thought to play an important role in masticatory stress

617 resistance (e.g., Picq \& Hylander, 1989; Hylander, Johnson \& Picq, 1991; Ravosa, 1991a,b; 
618 Ravosa et al., 2000) it is interesting to note that our human FEMs experienced higher von Mises

619 strain magnitudes than chimpanzees at all three of the supraorbital sites examined, particularly

620 during premolar biting. Between the human and chimpanzee samples, differences were found to

621 be greatest at the working and balancing dorsal orbitals, not the dorsal interorbital, supporting the

622 idea that the brow ridge cannot be modeled as a bent beam (Picq \& Hylander, 1989; see also

623 Chalk et al., 2011). The fact that the smaller brows of humans experienced elevated strain

624 magnitudes during biting could be interpreted as meaning that large brow ridges are an

625 adaptation to resist masticatory loads. However, a wealth of experimental data on humans and

626 non-human primate species has shown (e.g., Hylander, Johnson \& Picq, 1991; Ravosa et al.,

627 2000; Szwedowski, Fialkov \& Whyne, 2011; Ross et al., 2011; Maloul et al., 2012) that strains

628 along the supraorbital margin are relatively low during biting and chewing, which is supported

629 by the results presented here. Therefore, it is more reasonable to interpret differences in

630 supraorbital morphology between humans and chimpanzees as being related to some non-dietary

631 function, and that the resulting increases in brow ridge strain among humans are experienced as a

632 secondary byproduct. For example, Moss and Young (1966) suggest that a large separation is

633 formed posterior to the orbits when brain size is small, forming a supraorbital ridge. When brain

634 size is large, the frontal bone is more steeply inclined posterior to the orbits, forming a vertical

635 forehead rather than a large torus. A byproduct of this missing bar of bone above the orbits

636 among modern humans could be that strain magnitudes are mildly elevated in that region.

637 Overall, our findings show that the human craniofacial skeleton is weaker than that of

638 chimpanzees when subjected to feeding loads. These findings support the hypothesis that dietary

639 changes involving a shift to softer and/or more processed foods along the modern human lineage

640 has led to masticatory gracilization and reduced structural strength of the bony facial skeleton 
641 (e.g., Lieberman et al., 2004). However, in their biomechanical analysis, Wroe et al. (2010)

642 recently found that although the human cranium is less robust, it experiences low peak strains

643 and an even distribution of facial strain magnitudes compared to extant apes and fossil

644 australopith species. Differences between our results and those of Wroe et al. (2010) could

645 reflect differences in the way muscle loads were applied to the models in each analysis and/or the

646 manner in which models were constrained. For example, we applied both normal and tangential

647 tractions over entire muscle areas using Boneload (Grosse et al., 2007), whereas Wroe et al.

648 (2010) loaded their models with muscles modeled as straight pre-tensioned beam elements.

649 However, we conducted a sensitivity analysis to explore this possibility further (see

650 Supplementary Information) and found that these differences in methodology only resulted in

651 small differences in strain magnitude at most locations across the craniofacial skeleton.

652 Another possible explanation for the differences between our study and the study by

653 Wroe et al. (2010) relates to the magnitudes of the applied muscle forces. Wroe et al. (2010)

654 subjected their FEMs to three sets of simulated biting on various teeth. In their first simulation of

655 the three, FEMs were assigned a set of species-specific muscle forces (or muscle force estimates)

656 from the literature. In a second simulation, models were scaled to the surface area of their

657 chimpanzee model and re-loaded using chimpanzee muscle forces. Lastly, in the third

658 simulation, models were scaled to the surface area of their chimpanzee model and loaded with

659 muscle loads required to generate an equivalent bite force. In this third simulation, the high

660 biting leverage offered by the retracted human face meant that the forces required to generate a

661 bite compared to the other hominoids examined were relatively low. Therefore, Wroe et al.

662 (2010) concluded that the human facial skeleton may in fact be well-adapted to resist masticatory

663 stresses generated during high magnitude biting. Importantly, however, mean element von Mises 
664 stresses were found to be relatively high in their human FEM during the second simulation,

665 where FEMs were scaled to the same surface area and loaded with equivalent muscle forces.

666 This is the most similar of their three scaling procedures to the scaling performed here (scaling

667 muscle forces to model volume ${ }^{2 / 3}$ ), which we believe is the best means for removing the effects

668 of size on comparisons of mechanical performance (e.g., Dumont, Grosse \& Slater, 2009; Strait

669 et al., 2010).

670

671 Bite force production and efficiency: are humans suited to produce large biting forces?

672 When analyzed using human bone and muscle properties (i.e., ALL-HUM models), our

673 human FEMs produced bite forces of 333-507 $\mathrm{N}$ at the premolar $\left(\mathrm{P}^{3}\right)$ and $496-756 \mathrm{~N}$ at the molar

$674\left(\mathrm{M}^{2}\right)$. These results are similar to, but lower than, previous estimates of human bite force

675 production using both 2D and 3D modeling techniques (e.g., Wroe et al., 2010; Eng et al., 2013).

676 For example, using skeletal measurements and data on muscle cross-section, Eng et al. (2013)

677 recently estimated that humans are capable of producing approximately $660-1106 \mathrm{~N}_{\text {of }} \mathrm{M}^{2}$ bite

678 force, while Wroe et al. (2010) estimated a maximum unilateral $\mathrm{M}^{2}$ bite force of 1109-1317 N

679 using FEA. However, our $\mathrm{M}^{2}$ bite force results are bracketed by bite force transducer data

680 collected from various western populations, which range from approximately $368 \mathrm{~N}$ (Sinn, de

681 Assis \& Throckmorton, 1996) to around 911 N (Waltimo, Nystram \& Kananen 1994), although

682 Inuit males have been shown to produce an average of $1277 \mathrm{~N}$ in $\mathrm{M}^{2}$ bite force (Waugh, 1937).

683 Therefore, our results for bite force production lie within and do not exceed the known range of

684 in vivo variation exhibited by recent human populations.

685 Because chimpanzees have absolutely and relatively larger jaw adductor muscles than

686 humans (e.g., Taylor \& Vinyard, 2013), it is no surprise that the chimp FEMs were capable of 
687 producing more forceful bites than our human FEMs when loaded with species-specific muscle 688 forces (compare data in Table 9 to Smith et al., 2015b, Table 4). However, when loaded with 689 muscle forces scaled to remove differences in size (as in the CHIMPED model variants), we 690 found that humans are more efficient producers of bite forces, in terms of biting leverage, 691 consistent with the findings of Wroe et al. (2010). Specifically, the mechanical advantage (MA) 692 for $\mathrm{P}^{3}$ biting in humans ranged 0.39-0.47, compared to 0.32-0.42 in chimpanzees (Smith et al., 693 2015b), with only two chimps overlapping the human range. Humans were found to exhibit even 694 more elevated leverage during $\mathrm{M}^{2}$ biting (0.60-0.71), with only one individual overlapping the 695 chimpanzee range (0.49-0.61). When comparing these data using statistical analysis as a 696 heuristic guide, humans were found to be significantly more efficient at producing bite forces at 697 both mesial and distal bite points. The CHIMPED humans were even found to exhibit a biting 698 efficiency similar to that observed in australopiths (Smith et al., 2015a). In fact, $\mathrm{P}^{3} \mathrm{MA}$ in $P$. 699 boisei (0.40) and A. africanus (0.41) were near the lower end observed in humans. The FEM of 700 A. africanus also generated $\mathrm{M}^{2}$ bites with similar efficiency (0.62) to humans, whereas $P$. boisei

701

702

703

704

705

706

707

708

709

produced more mechanically efficient (0.75) molar bites (Smith et al., 2015a).

Our data on bite force efficiency in humans support previous findings that have demonstrated the mechanical advantage of modern human bony facial architecture compared to both non-modern humans and non-human primate species (e.g., Spencer \& Demes, 1993; O’Connor, Franciscus \& Holton, 2005; Lieberman, 2008, 2011; Wroe et al., 2010; Eng et al., 2013). Using estimates of muscle leverage from 2D measurements (Lieberman, 2008, 2011), humans have been shown to achieve high biting leverage through a marked degree of facial retraction (orthognathism), which reorients the muscles of mastication relative to the tooth rows. As noted above, we found that our human FEMs produced bite forces with leverage ratios 
710 similar to those observed in A. africanus and P. boisei (Smith et al., 2015a). However,

711 australopiths achieve high biting leverage through an anterior positioning of the chewing muscles

712 relative to the tooth rows (Rak, 1983; Strait et al., 2009, 2010; Smith et al., 2015a). In humans,

713 the midfacial region is "tucked" beneath the anterior cranial fossa (Lieberman, McBratney \&

714 Krovitz, 2002; Lieberman et al., 2004; Lieberman, 2008, 2011), which similarly places bite

715 points in a position that offers higher mechanical advantage to the jaw adductors.

716 Although the human cranium can theoretically produce mechanically efficient bite forces,

717 the production of unilateral molar $\left(\mathrm{M}^{2}\right)$ bite force is limited by the risk of temporomandibular

718 joint (TMJ) distraction, as predicted by the constrained lever model (Greaves, 1978; Spencer,

719 1998, 1999). Specifically, we found that all seven of the CHIMPED human FEMs experienced a

720 highly distractive (tensile) reaction force at the working-side joint during molar biting. These

721 forces have the effect of "pulling" the mandibular condyle from the jaw joint, increasing the risk

722 of joint dislocation (Spencer, 1998, 1999). As noted in the introduction, the soft tissues of the

723 mammalian jaw joint are well suited to resist compressive joint reaction forces, but are poorly

724 configured to resist distractive joint forces that "pull" the mandibular condyle from the cranial

725 base (Greaves, 1978; Spencer, 1998, 1999). In contrast, only one of the six chimpanzee FEMs

726 analyzed by Smith et al. (2015a) generated a tensile force at the working TMJ, and this reaction

727 was only very weakly tensile (12.7 N). Similarly, Smith et al. (2015b) found that their FEMs of

728 P. boisei and A. africanus lacked working-side distraction and were able to produce "stable"

729 bites on both the premolars and molars, offering these species the ability to produce maximally

730 forceful molar bites with limited risk of causing pain and/or damage to the TMJ capsule.

731 Interestingly, when loaded with human muscle forces (i.e., ALL-HUM), two of the

732 human FEMs (TIGA and WAFR) were capable of maintaining weakly compressive reaction 
733 forces at both TMJs during molar biting. Additionally, balancing side force reductions required

734 to eliminate distraction in the remaining models were proportionately less $(5-15 \%)$ than when

735 applying chimpanzee forces (15\%-30\%). Comparisons of the muscle loads applied to the models

736 and their force ratios in the ALL-HUM and CHIMPED models (see Tables 9 and 11) reveal that

737 chimpanzees devote a higher proportion of muscle strength to anteriorly-positioned muscle

738 compartments (superficial masseter and anterior temporalis) compared to more posteriorly-

739 positioned ones (deep masseter and medial pterygoid). Therefore, it is tempting to suggest that

740 changes in human jaw muscle force ratios may have coincided with the retraction of the lower

741 face during human evolution in order to reduce the risk of TMJ distraction. Likewise, if the

742 repositioning of cranial elements for reasons other than food processing (Lieberman, 2008;

743 Lieberman \& Zink, 2016) led to an increase in biting efficiency but the generation of working

744 side joint distraction during molar biting, the overall reduction of chewing muscle size in Homo

745 could also be viewed as a result of positive selection rather than relaxed selection so as to lessen

746 these distractive forces.

Our findings that humans are limited in their ability to produce forceful unilateral molar

bites are supported by data on bite force and muscle activity in humans. Spencer $(1995,1998)$

tested some predictions of the constrained lever model and found that humans produced bite

750 forces that increased as the bite point moved from the incisors to the first molar. Moving from

$751 \mathrm{M}^{1}$ to $\mathrm{M}^{3}$, bite forces were found to decrease as a result of the decreasing balancing force muscle

752 recruitment required to avoid joint distraction. Spencer (1995) also notes that most of the

753 participants (8 of 10) in his analysis reported pain near the working-side TMJ when biting

754 forcefully using the back molars. In addition to this study, Hylander (1977) suggests that

755 specialized anterior tooth biting and increased masticatory muscle leverage may be related to the 
756 high incidence of third molar reduction and agenesis among modern Inuit due to the increased

757 risk of distraction when biting on these teeth, although the results of our single pre-historic

758 Arctic FEM (TIGA) provide no support for this hypothesis. Similarly, Spencer (2003)

759 demonstrates that seed predating New World primates with adaptations for increased anterior

760 bite force have relatively small third molar roots.

761 As discussed above, Wroe et al. (2010) analyzed human feeding biomechanics within a

762 comparative context. One of the principal findings of their analysis, supported by the data

763 presented here, is that humans are capable of generating bite forces with higher mechanical

764 efficiency than chimpanzees. Wroe et al. use this as evidence to argue that human craniofacial

765 evolution may have been influenced by selection for powerful biting behaviors. However, the

766 results of this study showing the comparative weakness of the human cranium combined with the

767 increased risk of jaw joint distraction during molar biting leads us to interpret the increased

768 biting leverage exhibited by humans, which is particularly high among recent populations

769 (Spencer \& Demes, 1993; O’Connor, Franciscus \& Holton, 2005), to be a byproduct of human

770 facial orthognathism, which may be at least partly related to facial size reduction. Human facial

771 flatness may also have been acquired through selection for some non-dietary function. For

772 example, Lieberman $(2008,2011)$ suggests that the marked degree of facial retraction exhibited

773 by modern human crania could be related to changes in brain size and cranial base flexion.

774 However, Ross (2013) shows that basicranial flexion cannot produce significant facial retraction

775 on its own. Alternatively, Holton et al. (2010) propose that dietary shifts leading to reduced

776 facial strain magnitudes among early human species may have led to reduced facial growth and

777 earlier fusion of the maxillary sutures, and thus smaller and more retracted facial skeletons. 
Although the majority of the morphological and mechanical evidence is not consistent

779

780

781

782

783

784

785

786

787

788

789

790

791

792

793

794

795

796

797

798

799

800

with the hypothesis that the human masticatory apparatus has experienced recent selection for

high magnitude biting, the results of our analysis cannot reject the hypothesis that, in addition to changes in diet and tool use, increases in muscle force efficiency during human evolution could have led to relaxed selection for large chewing muscle size and reductions in facial size (Wroe et al., 2010) or that humans benefited from increased biting leverage when using submaximal forces by exerting less energy per bite. Our results for premolar biting leverage also do not conflict directly with the hypothesis that anterior tooth biting could have been selectively important in humans. However, the reduced size of the premolar teeth in humans increases the risk of tooth crown fracture (Constantino et al., 2010). Therefore, studies on premolar size and strength are not consistent with the hypothesis that humans are particularly well adapted for forcefully loading their anterior teeth, but such studies have yet to be conducted on incisors or canines, which are the more likely to be used during paramasticatory activities. For example, Hylander (1977) identifies features of the modern Inuit craniofacial skeleton that he argues to be adaptations for powerful biting behaviors using the incisors, although our single pre-historic Arctic FEM (TIGA) was not found to be exceptional in this regard. Additionally, Spencer \& Ungar (2000) show that incisor bite force leverage varies in relation to the intensity of incisor tooth use among some Native American populations. Similarly, it is possible that differences in anterior tooth use among "archaic" members of the genus Homo are reflected in mechanical differences between the species. In particular, the Neanderthals (H. neanderthalensis) exhibit a number of derived characteristics hypothesized to be adaptations for forceful incisor biting (e.g., Brace, 1962; Smith, 1983; Trinkaus, 1983, 1987; Rak, 1986; Demes, 1987). Notably, Spencer \& Demes (1993) show that Neanderthals exhibit high incisor bite force leverage relative to $H$. 
801 heidelbergensis (but not modern H. sapiens). In order to maintain functional use of the posterior

802 dentition (i.e., avoid TMJ distraction), Spencer \& Demes (1993) further show that the molar

803 tooth row in Neanderthals was anteriorly shifted, resulting in the characteristic retromolar gap.

804 Data on enamel thickness seemingly contrasts with the hypothesis that humans have 805 experienced relaxed selection for powerful biting behaviors. Specifically, a number of studies

806 find that recent human populations exhibit thick molar enamel (e.g., Martin, 1983, 1985;

807 Olejniczak et al., 2008; Smith et al., 2006; Vogel et al., 2008), which has been interpreted as a

808 primitive retention. However, notwithstanding disagreements over the significance of enamel

809 thickness (Grine, 2005), Smith et al. (2012) recently show that "thick" molar enamel in humans

810 is primarily the result of small coronal dentine areas. They found that enamel area in humans is

811 reduced, but there was a disproportionately large reduction in dentine to enamel as human teeth

812 were evolving smaller size, resulting in a relatively "thick" enamel cap. Thus, Smith et al. (2012)

813 argue that the dichotomy between thick and thin enamel is an oversimplification.

\section{CONCLUSIONS}

816 We examined the biomechanical consequences of human masticatory gracilization and

817 intraspecific variation within the constrained lever model of feeding biomechanics (Spencer,

818 1999) and tested the hypothesis that the human face is well configured to generate and withstand

819 high biting forces relative to chimpanzees. We found that our biomechanical models of human

820 crania were, on average, less structurally stiff than the crania of chimpanzees when assigned

821 equivalent bone properties, constraints, and physiologically-scaled muscle forces. These results

822 are consistent with the facial reduction exhibited by modern humans. We also found that modern

823 humans are efficient producers of bite force, consistent with previous analyses (Spencer \& 
824 Demes, 1993; O’Connor, Franciscus \& Holton, 2005; Lieberman, 2008, 2011; Wroe et al., 2010;

825 Eng et al., 2013), but that distractive (tensile) reaction forces are generated at the working

826 (biting) side jaw joint during $\mathrm{M}^{2}$ biting. In life, such a configuration would have increased the

827 risk of joint dislocation and constrained the maximum recruitment levels of the masticatory

828 muscles, meaning that the human cranium is poorly suited to produce forceful unilateral molar

829 bites. Our results do not conflict directly with the hypothesis that premolar biting could have

830 been selectively important in humans, although the reduced size of these teeth in humans has

831 been shown to increase the risk of tooth crown fracture. We interpret our results to suggest that

832 human craniofacial evolution was probably not driven by selection for high magnitude biting,

833 and that increased masticatory muscle efficiency in humans is likely to be a byproduct of

834 selection for some non-dietary function (Lieberman, 2008) or perhaps related to reduced

835 masticatory strain and sutural growth restrictions (Holton et al., 2010).

836 Our results provide support for the hypothesis that a shift to the consumption of less

837 mechanically challenging foods and/or the innovation of extra-oral food processing techniques

838 (e.g., stone tool use, cooking) along the lineage leading to modern Homo sapiens relaxed the

839 selective pressures maintaining features favoring forceful biting and chewing behaviors,

840 including large teeth and robust facial skeletons, leading to the characteristically small and

841 gracile faces of modern humans (e.g., Brace, Smith \& Hunt, 1991; Wrangham et al., 1999;

842 Lieberman et al., 2004; Ungar et al., 2006a,b; Wood, 2009). To contribute to our further

843 understanding, future studies should aim to identify the ecological changes that may have led to

844 the emergence of such shifts in dietary behavior. Were these changes initiated by changes in

845 climate, competition, resource availability, or some combination of these factors? To what extent

846 is craniofacial gracilization part of a general pattern of skeletal gracilization in humans (Ruff et 
847 al, 1993, 2015; Chirchir et al, 2015; Ryan \& Shaw, 2015)? These questions will be addressed by

848 gaining further insight into the dietary ecology and feeding adaptations of species near the

849 origins of the modern human lineage through work on biomechanics, paleoecology, archaeology,

850 bone chemistry, and dental wear, each of which inform key components necessary to obtaining a

851 more complete understanding of human craniofacial evolution.

852

853 ACKNOWLEDGEMENTS

854 We thank Gisselle Garcia-Pack and Kristen Mable of the AMNH for access to human skeletal 855 collections. We also thank Tim Ryan and Tim Stecko of the Center for Quantitative Imaging at 856 Penn State for assistance in acquiring CT image data of modern human crania.

857

858

\section{REFERENCES}

859 Agrawal KR, Lucas PW, Prinz JF, Bruce IC. 1997. Mechanical properties of foods responsible 860 for resisting food breakdown in the human mouth. Arch Oral Biol 42:1-9.

Arbel G, Hershkovitz I, Gross MD. 2000. Strain distribution on the skull due to occlusal loading: an anthropological perspective. Homo 51:30-55.

Baab KL, Freidline SE, Wang SL, Hanson T. 2010. Relationship of cranial robusticity to cranial form, geography and climate in Homo sapiens. Am J Phys Anthropol 141:97-115.

Benninghoff A. 1925. Spaltlinien am Knochen, ein method zur ermittlung der architektur platter kochen. Verh Anat Ges 34:189-206.

Berger LR, de Ruiter DJ, Churchill SE, Schmid P, Carlson KJ, Dirks PHGM, Kibii JM. 2010. Australopithecus sediba: a new species of Homo-like australopith from South Africa. Science 328:195-204. 
870 Bluntschli H. 1926. Rückwirkung des Kieferapparates auf den Gesamtschadel. Z zahnarztl $871 \quad$ Orthopäd 18:57-59.

872 Bookstein FL. 1991. Morphometric tools for landmark data: geometry and biology. Cambridge: 873 Cambridge University Press.

874 Brace CL. 1962. Cultural factors in the evolution of the human dentition. In: Montague MFA, 875 editor. Culture and the evolution of man. Oxford: Oxford University Press. p 343-354. 876 Brace C, Smith SL, Hunt KD. 1991. What big teeth you had grandma! Human tooth size, past and present. Advances in Dental Anthropology 33-57.

878 Carmody RN, Wrangham RW. 2009. The energetic significance of cooking. J Hum Evol 57:379$879 \quad 391$.

880 Carmody RN, Weintraub GS, Wrangham RW. 2011. Energetic consequences of thermal and 881 nonthermal food processing. . Proc Natl Acad Sci USA 108:19199-19203.

882 Chalk J, Richmond BG, Ross CF, Strait DS, Wright BW, Spencer MA, Wang Q, Dechow PC. 883 2011. A finite element analysis of masticatory stress hypotheses. Am J Phys Anthropol. $145: 1-10$.

885

886

887

888

889

890

891

892

Chirchir H, Kivell TL, Ruff CB, Hublin J-J, Carlson K, Zipfel B, Richmond BG. 2015. Recent origin of low trabecular bone density in modern humans. Proc Natl Acad Sci USA 112:366-371.

Constantino PJ, Lee JJ-W, Chai H, Zipfel B, Ziscovici C, Lawn BR, Lucas PW. 2010. Tooth chipping can reveal the diet and bite forces of fossil hominins. Biol Lett 6:826-829.

Curtis N, Witzel U, Fitton L, O’Higgins P, Fagan M. 2011. The mechanical significance of the temporal fasciae in Macaca fascicularis: an investigation using finite element analysis.

Davis JL, Dumont ER, Strait DS, Grosse IR. 2011. An efficient method of modeling 
893

894

895

896

897

898

899

900

901

902

903

904

905

906

907

908

909

910

911

912

913

914 Fleagle JG, Gilbert CC, Baden AL. 2010. Primate cranial diversity. Am J Phys Anthropol

915

material properties using a thermal diffusion analogy: an example based on craniofacial bone. PLoS ONE 6:e17004.

Demes B. 1987. Another look at an old face: biomechanics of the neandertal facial skeleton reconsidered. J Hum Evol 16:297-303.

Demes B, Creel N. 1988. Bite force, diet, and cranial morphology of fossil hominids. J Hum Evol 17:657-670.

Dumont ER, Grosse IR, Slater GJ. 2009. Requirements for comparing the performance of finite element models of biological structures. J Theor Biol 256:96-103.

Eisenberg NA, Brodie AG. 1965. Antagonism of temporal fascia to masseteric contraction. Anat Rec 152:185-192.

Ellis E, El-Attar A, Moos KF. 1985. An analysis of 2,067 cases of zygomatico-orbital fracture. J Oral Maxillofac Surg 43:417-428.

Ellis E. 2012. Chapter 16: Fractures of the zygomatic complex and arch. In: Fonseca RJ, Barber HD, Powers MP, Frost DE, editors. Oral and Maxillofacial Trauma. St. Louis, MO: Saunders. p 354-415.

Endo B. 1965. Distribution of stress and strain produced in the human facial skeleton by masticatory force. J Anthropol Soc Nippon 73:123-136.

Endo B. 1966. Experimental studies on the mechanical significance of the form of the human facial skeleton. J Faculty of Sci 3:1-106.

Eng CM, Lieberman DE, Zink KD, Peters MA. 2013. Bite force and occlusal stress production in hominin evolution. Am J Phys Anthropol 151:544-557. $142: 565-578$. 
916 Görke O. 1904. Beitrag zur funktionellen gestaltung des schadels bei den anthropomorphen und

917 menschen durch untersuchung mit rontgenstrahlen. Arch Anthrop 1:91-108.

918 Greaves WS. 1978. The jaw lever system in ungulates: a new model. J Zool 184:271-285.

919 Grine FE. 2005. Enamel thickness of deciduous and permanent molars in modern Homo sapiens. $920 \quad$ Am J Phys Anthropol. 126:14-31.

921 Groopman EE, Carmody RN, Wrangham RW. 2015. Cooking increases net energy gain from a lipid-rich food. AM J Phys Anthropol 156:11-18.

923 Grosse IR, Dumont ER, Coletta C, Tolleson A. 2007. Techniques for modeling muscle induced forces in finite element models of skeletal structures. Anat Rec 290A:1069-1088.

Holton NE, Franciscus RG, Nieves MA, Marshall SD, Reimer SB, Southard, TE, Keller JC, Maddux SD. 2010. Sutural growth restriction and modern human facial evolution: an experimental study in a pig model. J Anat 216:48-61.

928

929

930

931

932

933

934

935

936

937

938

Hylander WL. 1977. The adaptive significance of Eskimo craniofacial morphology. In: Dahlberg AA, Graber TM, editors. Orofacial growth and development. Chicago, IL: Aldine Publishing Company. p 129-169.

Hylander WL, Johnson KR. 1997. In vivo bone strain patterns in the zygomatic arch of macaques and the significance of these patterns for functional interpretations of craniofacial form. Am J Phys Anthropol 102:203-232.

Hylander WL, Johnson KR, Picq PG. 1991. Masticatory-stress hypotheses and the supraorbital region of primates. Am J Phys Anthropol 86:1-36.

Keyak JH, Rossi SA. 2000. Prediction of femoral fracture load using finite element models: an examination of stress- and strain-based failure theories. J Biomech 33:209-214.

Kimbel WH, Rak Y, Johanson DC. 2004. The skull of Australopithecus afarensis. New York: 
Oxford University Press.

940 Ledogar JA. 2015. Human feeding biomechanics: intraspecific variation and evolution. Ph.D

941 dissertation, University at Albany.

942 Lieberman DE. 2008. Speculations about the selective basis for modern human craniofacial 943 form. Evol Anthropol 17:55-68.

944 Lieberman DE. 2011. The evolution of the human head. Cambridge, MA: Belknap Press.

945 Lieberman DE, McBratney BM, Krovitz GE. 2002. The evolution and development of cranial 946 form in Homo sapiens. Proc Natl Acad Sci USA 99:1134-1139.

947 Lieberman DE, Krovitz G, Devlin M, Yates F, St. Clair M. 2004. Effects of food processing on 948 masticatory strain and craniofacial growth in a retrognathic face. J Hum Evol 46:655-677.

949 Lucas PW. 2004. Dental function morphology: how teeth work. Cambridge: Cambridge $950 \quad$ University Press.

951 Lucas PW, Luke DA. 1984. Optimum mouthful for food comminution in human mastication. 952 Arch Oral Biol 29:205-210.

953 Maloul A, Fialkov J, Whyne C. 2011. The impact of voxel size-based inaccuracies on the 954 mechanical behavior of thin bone structures. Ann Biomed Eng 39:1092-1100.

Maloul A, Regev E, Whyne CM, Beek M, Fialkov JA. 2012. In vitro quantification of strain 956 patterns in the craniofacial skeleton due to masseter and temporalis activities. J Craniofac

957 Surg 23:1529-1534.

958 Martin LB. 1983. Relationships of the later Miocene Hominoidea. Ph.D dissertation, 959 Unversity College London.

960 Martin LB. 1985. Significance of enamel thickness in hominoid evolution. Nature 314:260-263.

961 Moss ML, Young RW. 1960. A functional approach to craniology. Am J Phys Anthropol 
963 Mundry R, Fischer J. 1998. Use of statistical programs for nonparametric tests of small samples 964 often leads to incorrect p-values: examples from animal behavior. Anim Behav 56:256965259.

966 Murphy RA. 1998. Skeletal muscle. In: Berne RM, Levy MN, editors. Physiology. St. Louis, 967 MO: Mosby. p 294.

968 Nagasao T, Nakajima T, Kimura A, Kaneko T, Jin H, Tamaki T. 2005. The dynamic role of 969 buttress reconstruction after maxillectomy. Plast Reconstr Surg 115:1138-1349.

970 O’Connor CF, Franciscus RG, Holton NE. 2005. Bite force production capability and efficiency 971 in neandertals and modern humans. Am J Phys Anthropol 127:129-151.

972 Olejniczak AJ, Smith TM, Feeney RN, Macchiarelli R, Mazurier A, Bondioli L, Rosas A, Fortea 973 J, del la Rasilla M, Garcia-Tabernero A, Radovcic J, Skinner MM, Toussaint M, Hublin J-J. 2008. Dental tissue proportions and enamel thickness in Neandertal and modern human molars. J Hum Evol 55:12-23.

Organ C, Nunn CL, Machanda Z, Wrangham RW. 2011. Phylogenetic rate shifts in feeding time during the evolution of Homo. Proc Natl Acad Sci USA 108:14555-14559.

Peterson J, Dechow PC. 2002. Material properties of the inner and outer cortical tables of the human parietal bone. Anat Rec 268:7-15.

Picq PG, Hylander WL. 1989. Endo's stress analysis of the primate skull and the functional significance of the supraorbital region. Am J Phys Anthropol 79:393-398.

Pollock RA. 2012. Craniomaxillofacial buttresses: anatomy and operative repair. New York: Thieme Medical Publishers. 
984 Popowics TE, Herring SW. 2007. Load transmission in the nasofrontal suture of the pig, Sus 985 scrofa. J Biomech 40:837-844.

986 Rak Y. 1983. The australopithecine face. New York: Academic Press.

987 Rak Y. 1986. The Neanderthal face: a new look at an old face. J Hum Evol 15:151-164.

988 Ravosa MJ. 1991a. Ontogenetic perspective on mechanical and non-mechanical models of 989 primate circumorbital morphology. Am J Phys Anthropol 85: 95-112.

990 Ravosa MJ. 1991b. Interspecific perspective on mechanical and nonmechanical models of 991 primate circumorbital morphology. Am J Phys Anthropol 86: 369-396.

992 Ravosa MJ, Noble VE, Hylander WL, Johnson KR, Kowalski EM. 2000. Masticatory stress, 993 994 orbital orientation and the evolution of the primate postorbital bar. J Hum Evol 38:667693.

995

996

997

998 999

1000

1001

1002

1003

1004

1005

1006

Robinson JT. 1954. Prehominid dentition and hominid evolution. Evolution (N Y) 8:325-334.

Ross CF. 2001. In vivo function of the craniofacial haft: the interorbital "pillar." Am J Phys Anthropol 116:108-139.

Ross CF. 2013. Complexity, modularity, and integration in the human head. J Hum Evol 64:5667.

Ross CF, Iriarte-Diaz J. 2014. What does feeding system morphology tell us about feeding? Evol Anthropol 23:105-120.

Ross CF, Patel BA, Slice DE, Strait DS, Dechow PC, Richmond BG, Spencer MA. 2005. Modeling masticatory muscle force in finite-element analysis: sensitivity analysis using principal coordinates analysis. Ant Rec 283A:288-299.

Ross CF, Berthaume MA, Dechow PC, Iriate-Diaz J, Porro LB, Richmond BG, Spencer M, Strait DS. 2011. In vivo bone strain and finite-element modeling of the craniofacial haft 
in catarrhine primates. J Anat 218:112-141.

1008

1009

1010

1011

1012

1013

1014

1015

1016

1017

1018

1019

1020

1021

1022

1023

1024

1025

1026

1027

1028

1029

Ruff CB, Trinkaus E, Walker AC, Larsen CS. 1993. Postcranial robusticity in Homo. I:

Temporal

trends and mechanical interpretation. Am J Phys Anthropol. 91: 21-53.

Ruff CB, Holt B, Niskanen M, Sladek V, Berner M, Garofalo E, Garvin HM, Hora M, Junno JA, Schuplerova E, Vilkama R, Whittey E. 2015. Gradual decline in mobility with the adoption of food production in Europe. Proc Natl Acad Sci USA 112:7147-7152.

Ryan TM, Shaw CN. 2015. Gracility of the modern Homo sapiens skeleton is the result of decreased biomechanical loading. Proc Natl Acad Sci USA 112:372-377.

Schwartz-Dabney CL, Dechow PC. 2002. Accuracy of elastic property measurement in mandibular cortical bone is improved by using cylindrical specimens. J Biomech Eng 124:714-723.

Sinn DP, de Assis EA, Throckmorton GS. 1996. Mandibular excursions and maximum bite forces in patients with temporomandibular joint disorders. J Oral Maxillofac Surg 54:671-679.

Skelton RR, McHenry HM. 1992. Evolutionary relationships among early hominids. J Hum Evol 23:309-349.

Slice DE, ed. 2005. Modern morphometrics in physical anthropology, vol. 6. Springer Science \& Business Media.

Smith FH. 1983. Behavioral interpretations of changes in craniofacial morphology across the archaic/modern Homo sapiens transition. In. Trinkaus E, editor. The Mousterian legacy. BAR. p. 141-163. 
1030 Smith AL, Benazzi S, Ledogar JA, Tamvada K, Pryor Smith LC, Weber GW, Spencer MA,

1031 Lucas PW, Michael S, Shekeban A, Al-Fadhalah K, Almusallam AS, Dechow PC,

1032

1033

Groasse IR, Ross CF, Madden RH, Richmond BG, Wright BW, Wang Q, Byron C, Slice

1034

DE, Wood S, Dzialo C, Berthaume MA, van Casteren A, Strait DS. 2015a. The feeding biomechanics and dietary ecology of Paranthropus boisei. Ant Rec 298:145-167.

1035

1036

1037

1038

1039

1040

1041

1042

1043

1044

1045

1046

1047

1048

1049

1050

1051

1052

Smith AL, Benazzi S, Ledogar JA, Tamvada K, Pryor Smith LC, Weber GW, Spencer MA, Dechow PC, Grosse IR, Ross CF, Richmond BG, Wright BW, Wang Q, Byron C, Slice DE, Strait DS. 2015b. Biomechanical implications of intraspecific shape variation in chimpanzee crania: moving towards an integration of geometric morphometrics and finite element analysis. Anat Rec 298:122-144.

Smith TM, Olejniczak AJ, Reid DJ, Ferrell RJ, Hublin JJ. 2006. Modern human molar enamel thickness and enamel-dentine junction shape. Arch Oral Biol 51:974-995.

Smith TM, Olejniczak AJ, Zermeno JP, Tafforeau P, Skinner MM, Hoffman A, Radovcic J, Toussaint M, Kruszynski R, Menter C, Moggi-Cecchi J, Glasmacher UA, Kullmer O, Schrenk F, Stringer C, Hublin J-J. 2012. Variation in enamel thickness within the genus Homo. J Hum Evol 62:395-411.

Spencer MA. 1998. Force production in the primate masticatory system: electromyographic tests of biomechanical hypotheses. J Hum Evol 34:25-54.

Spencer MA. 1999. Constraints on masticatory system evolution in anthropoid primates. Am J Phys Anthropol 108:483-506.

Spencer MA, Demes B. 1993. Biomechanical analysis of masticatory system configuration in Neandertals and Inuits. Am J Phys Anthropol 91:1-20.

Spencer MA, Ungar PS. 2000. Craniofacial morphology, diet and incisor use in three native 
American populations. Inter J Osteoarchaeol 10:229-241.

1054

1055

1056

1057

1058

1059

1060

1061

1062

1063

1064

1065

1066

1067

1068

1069

1070

1071

1072

1073

1074

1075

Spencer MA. 2003. Tooth-root form and function in platyrrhine seed-eaters. Am J Phys Anthropol 122:325-335.

Strait DS, Grine FE. 2004. Inferring hominoid and early hominid phylogeny using craniodental characters: the role of fossil taxa. J Hum Evol 47:399-452.

Strait DS, Grine FE, Moniz MA. 1997. A reappraisal of early hominid phylogeny. J Hum Evol $32: 17-82$.

Strait DS, Wang O, Dechow PC, Ross CF, Richmond BG, Spencer MA, Patel BA. 2005. Modeling elastic properties in finite element analysis: how much precision is needed to produce and accurate model? Anat Rec 283A:275-287.

Strait DS, Weber GW, Neubauer S, Chalk J, Richmond BG, Lucas PW, Spencer MA, Schrein C, Dechow PC, Ross CF, Grosse IR, Wright BW, Constantino P, Wood BA, Lawn B, Hylander WL, Wang Q, Byron C, Slice DE, Smith AL. 2009. The feeding biomechanics and dietary ecology of Australopithecus africanus. Proc Natl Acad Sci USA 106:2124-2129.

Strait DS, Grosse IR, Dechow PC, Smith AL, Wang QW, Weber GW, Neubauer S, Slice DE, Chalk J, Richmond BG, Lucas PW, Spencer MA, Schrein C, Wright BW, Byron C, Ross CF. 2010. The structural rigidity of the cranium of Australopithecus africanus: implications for diet, dietary adaptations, and the allometry of feeding biomechanics. Anat Rec 298:583-593.

Strait DS, Constantino P, Lucas PW, Richmond BG, Spencer MA, Dechow PC, Ross CF, Grosse IR, Wright BW, Wood BA, Weber GW, Wang Q, Byron C, Slice DE, Chalk J, Smith AL, Smith LC, Wood S, Berthaume M, Benazzi S, Dzialo C, Tamvada K, 
1076

1077

1078

1079

1080

1081

1082

1083

1084

1085

1086

1087

1088

1089

1090

1091

1092

1093

1094

1095

1096

1097

1098

Ledogar JA. 2013. Diet and dietary adaptations in early hominins: the hard food perspective. Am J Phys Anthropol 151:339-355.

Szwedowski TD, Fialkov F, Whyne CM. 2011. Sensitivity analysis of a validated subjectspecific finite element model of the human craniofacial skeleton. Proc Inst Mech Eng H 225:58-67.

Taylor AB, Vinyard CJ. 2013. The relationships among jaw-muscle fiber architecture, jaw morphology, and feeding behavior in extant apes and modern humans. Am J Phys Anthrpol 151:120-134.

Toro-Ibacache V, Zapata Muñoz V, O’Higgins P. 2015. The relationship between skull morphology, masticatory muscle force and cranial skeletal deformation during biting.

Toro-Ibacache V, Fitton LC, Fagan MJ, O'Higgins P. 2015. Validity and sensitivity of a human cranial finite element model: implications for comparative studies of biting performance.

Trinkaus E. 1983. Neanderthal postcrania and the adaptive shift to modern humans. In: The Mouseterian legacy: human biocultural change in the Upper Pleistocene. p 165-200. BAR.

Trinkaus E. 1987. The Neandertal face: evolutionary and functional perspectives on a recent hominid face. J Hum Evol 16:429-443.

Ungar P. 2012. Dental evidence for the reconstruction of diet in African early Homo. Curr Anthropol 53 S318-S329.

Ungar PS, Grine FE, Teaford MF. 2006. Diet in early Homo: a review of the evidence and a new model of adaptive versatility. Ann Rev Anthropol 35:209-228.

van Eijden TMGJ, Korfage JAM, Brugman P. 1997. Architecture of the human jaw-closing and jaw-opening muscles. Anat Rec 248:464-474. 
1099 Vogel ER, van Woerden JT, Lucas PW, Atmoko SSU, van Schaik CP, Dominy NJ. 2008.

1100

1101

1102

1103

1104

1105

1106

1107

1108

1109

1110

1111

1112

1113

1114

1115

1116

1117

1118

1119
Functional ecology and evolution of hominoid molar enamel thickness: Pan troglodytes schweinfurthii and Pongo pygmaeus wurmbii. J Hum evol 55:60-74.

Walker AC. 1991. The origin of genus Homo. In: Osawa S, Honjo T, editors. Evolution of Life: Fossils, Molecules, and Culture. Tokyo: Springer-Verlag. p 379-389.

Waltimo A, Nystram M, Kananen M. 1994. Bite force and dentofacial morphology in men with severe dental attrition. E J Oral Sci 102:92-96.

Wang Q, Dechow PC. 2006. Elastic properties of external cortical bone in the craniofacial skeleton of the rhesus monkey. Am J Phys Anthropol 131:402-415.

Wang Q, Strait DS, Dechow PC. 2006. A comparison of cortical elastic properties in the craniofacial skeletons of three primate species and its relevance to human evolution. $\mathbf{J}$ Hum Evol 51:375-382.

Wang Q, Wood SA, Grosse IR, Ross CF, Zapata U, Byron CD, Wright BW, Strait DS. 2012. The role of sutures in biomechanicanical dynamic simulation of a macaque cranial finite element model: implications for the evolution of craniofacial form. Anat Rec $295: 278-288$.

Waugh L. 1937. Dental observations among Eskimos. J Dent Res 16:355-356.

Weijs WA, Hillen B. 1984. Relationships between masticatory muscle cross-section and skull shape. J Dent Res 63:1154-1157.

Wood BA. 1992. Origin and early evolution of genus Homo. Nature 355:783-790. 
1120 Wood BA. 2009. "Where does the genus Homo begin, and how would we know?" The first

1121

1122

1123

1124

1125

1126

1127

1128

1129

1130

1131

1132 humans: origins of the genus Homo. In: Grine FE, Fleagle JG, Leakey RE, editors. New York: Springer. p 17-28.

Wrangham RW. 2009. Catching fire: how cooking made us human. New York: Basic Books.

Wrangham RW, Jones JH, Laden G, Pilbeam D, Conklin-Brittain N. 1999. The raw and the stolen: cooking and the ecology of human origins. Curr Anthropol 40:567-594.

Wroe S, Ferrara TL, McHenry CR, Curnoe D, Chamoli U. 2010. The craniomandibular mechanics of being human. Proc R Soc Lond B Biol Sci 277:3579-3586.

Zink KD, Lieberman DE. 2016. Impact of meat and Lower Palaeolithic food processing techniques on chewing in humans. Nature (Early View). doi:10.1038/nature16990.

Zioupos P, Smith CW, Yuehuei HA. 2000. Factors affecting mechanical properties of bone. In: An YH, Draughn RA (editors). Mechanical testing of bone and the bone-implant interface. Boca Raton: CRC Press. p 65-85. 


\section{Table $\mathbf{1}$ (on next page)}

Landmarks used in the geometric morphometric analysis of human craniofacial shape.

Coordinate data on these landmarks were collected by Baab and colleagues (Baab, 2007; Baab et al., 2010). The landmarks chosen for the analysis performed here are a subset of those used by Baab and colleagues, consisting mainly of facial landmarks. Landmark numbers and descriptions correspond to those in Baab (2007). 


\begin{tabular}{llll}
\hline Landmark & Number $^{\mathbf{1}}$ & Landmark & Number $^{\mathbf{1}}$ \\
\hline Alare (R, L) & 13,40 & Lingual canine margin (R, L) & 124,115 \\
Alveolare & 11 & M1-M2 contact (R, L) & 119,128 \\
Anterior nasal spine & 10 & M2-M3 contact (R, L) & 120,129 \\
Anterior pterion (R, L) & 24,51 & Malar root origin (R, L) & 31,58 \\
Basion & 67 & Mid post-toral sulcus & 6 \\
Bregma & 5 & Midline anterior palatine & 70 \\
Canine-P3 contact (R, L) & 116,125 & Mid-torus inferior (R, L) & 21,48 \\
Center of mandibular fossa (R, L) & 97,103 & Mid-torus superior (R, L) & 22,49 \\
Dacryon (R, L) & 16,43 & Nasion & 8 \\
Distal M3 (R, L) & 121,130 & Opisthion & 66 \\
Frontomalare orbitale (R, L) & 20,47 & Orbitale (R, L) & 18,45 \\
Frontomalare temporale (R, L) & 19,46 & P3-P4 contact (R, L) & 117,126 \\
Frontosphenomalare (R, L) & 23,50 & P4-M1 contact (R, L) & 118,127 \\
Frontotemporale (R, L) & 35,62 & Porion (R, L) & 27,54 \\
Glabella & 7 & Postglenoid (R, L) & 94,100 \\
Hormion & 68 & Rhinion & 9 \\
Incisivon & 71 & Root of zygomatic process (R, L) & 32,59 \\
Inferior entoglenoid (R, L) & 95,101 & Spheno-palatine suture (R, L) & 108,112 \\
Inferior zygotemporal suture (R, L) & 72,78 & Staphylion & 69 \\
Infraorbital foramen (R, L) & 12,39 & Stephanion (R, L) & 34,61 \\
Inion & 1 & Superior zygotemporal suture (R, L) & 25,52 \\
Jugale (R, L) & 26,53 & Supraorbital notch (R, L) & 17,44 \\
Lambda & 3 & Temporo-sphenoid suture (R, L) & 109,113 \\
Lateral articular fossa (R, L) & 96,102 & Zygomaxillare (R, L) & 14,41 \\
Lateral prosthion (R, L) & 114,123 & Zygoorbitale (R, L) & 15,42 \\
\hline
\end{tabular}


Table 2 (on next page)

Geographic distribution of human specimens included in the analysis of craniofacial shape variation.

All specimens are housed at the American Museum of Natural History (AMNH). 


\begin{tabular}{lr}
\hline Region/Population & N \\
\hline Aboriginal Australian & 9 \\
Khoe-San, South Africa & 3 \\
China & 6 \\
East Africa & 7 \\
Grand Gulch, Utah & 10 \\
Greifenberg, Carinthia, Austria & 6 \\
Heidenheim, Germany & 1 \\
Kakoletri, Peloponnesus, Greece & 1 \\
Maori, Waitakeri, New Zealand & 4 \\
Mongolia & 1 \\
Point Hope, Alaska & 12 \\
Southeast Asia & 12 \\
Tarnapol, Galicia, Poland & 2 \\
Tasmanian & 4 \\
Tierra del Fuego, Argentina & 3 \\
West Africa & 7 \\
\hline
\end{tabular}

1 


\section{Table 3 (on next page)}

Human crania sorted by their Euclidean distance from the group centroid.

The first 25 specimens represent the most distant from the group centroid, whereas the bottom row represents an "average" representative of human cranial shape based on its close proximity to the centroid. Values in parentheses represent the distances expressed in units of the mean pairwise distance $(0.068)$, which provides information on how much farther a particular cranium is from the centroid than the mean distance. Specimens are coded here following American Museum of Natural History (AMNH) catalog numbers. 


\begin{tabular}{llr}
\hline Specimen & Region/Population & Distance from centroid \\
\hline VL/2463 & Khoe-San, South Africa & $0.1011(1.49)$ \\
VL/3878 & Greifenberg, Austria & $0.0939(1.38)$ \\
$99 / 7889^{1}$ & Malay Archipelago, SE Asia & $0.0918(1.35)$ \\
VL/3818 & Greifenberg, Austria & $0.0885(1.31)$ \\
VL/269 & Tasmanian & $0.0881(1.30)$ \\
VL/229 & Kalmuk, Western Mongolia & $0.0876(1.29)$ \\
VL/408 & Mhehe, East Africa & $0.0871(1.28)$ \\
$99.1 / 511^{1}$ & Point Hope, Alaska & $0.0871(1.28)$ \\
$99 / 8155$ & Aboriginal Australian & $0.0842(1.24)$ \\
$99 / 6562$ & Māori, New Zealand & $0.0830(1.22)$ \\
VL/271 & Tasmanian & $0.0824(1.22)$ \\
VL/24701 & Khoe-San, South Africa & $0.0788(1.16)$ \\
VL/1902 & Māori, New Zealand & $0.0777(1.15)$ \\
$99.1 / 490$ & Point Hope, Alaska & $0.0770(1.14)$ \\
$99 / 8165$ & Aboriginal Australian & $0.0767(1.13)$ \\
VL/272 & Tasmanian & $0.0750(1.11)$ \\
VL3619 & Greifenberg, Austria & $0.0745(1.10)$ \\
$99 / 7333$ & Grand Gulch, Utah & $0.0741(1.09)$ \\
$99 / 8177$ & Aboriginal Australian & $0.0740(1.09)$ \\
VL/2267 & Kakoletri, Greece & $0.0733(1.08)$ \\
VL/1729 & Tientsin, China & $0.0728(1.07)$ \\
VL/1602 & Ashanti, West Africa & $0.0727(1.07)$ \\
VL/274 & Tasmanian & $0.0721(1.06)$ \\
VL/2389 & Ashanti, West Africa & $0.0721(1.06)$ \\
$99 / 8171$ & Aboriginal Australian & $0.0720(1.06)$ \\
\hline $99 / 7365^{1}$ & Grand Gulch, Utah & $0.0496(0.73)$ \\
\hline
\end{tabular}

${ }^{1}$ Specimens selected to be modeled using FEA. 


\section{Table 4 (on next page)}

Pairwise distances between the 6 human cranial specimens selected for use in finite element analysis.

Values in parentheses represent the distances expressed in units of the mean pairwise distance (0.068). Specimens are coded here following American Museum of Natural History (AMNH) catalog numbers. 


\begin{tabular}{lcccccc}
\hline & $\mathbf{V L} / \mathbf{2 4 6 3}$ & $\mathbf{V L / 3 8 7 8}$ & $\mathbf{9 9 / 7 8 8 9}$ & $\mathbf{9 9 . 1 / 5 1 1}$ & $\mathbf{V L} / \mathbf{2 4 7 0}$ & $\mathbf{V L} / \mathbf{1 6 0 2}$ \\
\hline $\mathbf{V L} / \mathbf{2 4 6 3}$ & $0.1634(1.70)^{1}$ & $0.0938(0.97)$ & $0.1534(1.59)^{1}$ & $0.1083(1.12)$ & $0.1145(1.19)$ \\
VL/3878 & & $0.1469(1.52)$ & $0.1304(1.35)$ & $0.1230(1.28)$ & $0.1385(1.44)$ \\
$\mathbf{9 9 / 7 8 8 9}$ & & & $0.1526(1.58)^{1}$ & $0.1178(1.22)$ & $0.1029(1.09)$ \\
$\mathbf{9 9 . 1 / 5 1 1}$ & & & & $0.1330(1.38)$ & $0.1256(1.30)$ \\
VL/2470 & & & & & $0.1049(1.09)$ \\
VL/1602 & & & & & \\
\hline
\end{tabular}

1 These represent the greatest pairwise distances in the final sample.

2 


\section{Table 5 (on next page)}

Muscle force scaling for the ALL-HUM and CHIMPED models of modern human crania.

Muscle forces in Newtons ( $\mathrm{N}$ ) were scaled by model size, where size is represented by model volume in $\mathrm{mm}^{3}$. Models are shown here ordered from smallest to largest in size. AT = anterior temporalis, $\mathrm{SM}=$ superficial masseter, $\mathrm{DM}=$ deep masseter, $\mathrm{MP}=$ medial pterygoid. 


\begin{tabular}{llcccccc}
\hline & & & & \multicolumn{4}{c}{ Muscle Force $(\mathbf{N})$} \\
\cline { 5 - 8 } Variant & Model & Volume $\left(\mathbf{m m}^{\mathbf{3}}\right)$ & Volume $^{\mathbf{2} / 3}$ & AT & SM & DM & MP \\
\hline ALL-HUM & KSAN2 & 331466 & 4789.53 & 128.41 & 105.15 & 53.29 & 108.64 \\
& MALP & 364129 & 5099.22 & 136.72 & 111.95 & 56.73 & 115.67 \\
& KSAN2 & 433331 & 5726.38 & 153.53 & 125.72 & 63.71 & 129.89 \\
& WAFR & 475555 & 6092.57 & 163.35 & 133.75 & 67.79 & 138.20 \\
& BERG & 489588 & 6211.84 & 166.55 & 136.37 & 69.11 & 140.90 \\
& GRGL & 557223 & 6771.52 & 181.55 & 148.66 & 75.34 & 153.60 \\
CHIMPED & TIGA & 655320 & 7544.59 & 202.28 & 165.63 & 83.94 & 171.14 \\
& KSAN2 & 331466 & 4789.53 & 556.13 & 572.02 & 85.07 & 189.02 \\
& MALP & 364129 & 5099.22 & 592.09 & 609.00 & 90.57 & 201.24 \\
& KSAN2 & 433331 & 5726.38 & 664.91 & 683.90 & 101.71 & 225.99 \\
& WAFR & 475555 & 6092.57 & 707.43 & 727.64 & 108.22 & 240.44 \\
& BERG & 489588 & 6211.84 & 721.28 & 741.88 & 110.34 & 245.15 \\
& GRGL & 557223 & 6771.52 & 786.26 & 808.73 & 120.28 & 267.24 \\
& TIGA & 655320 & 7544.59 & 876.02 & 901.05 & 134.01 & 297.74 \\
\hline
\end{tabular}

1 


\section{Table 6(on next page)}

Results of in vitro validation analysis.

Average values and standard deviations for maximum (MaxPrin) and minimum (MinPrin) principal strain magnitudes recorded during three in vitro loading trials on the left $\mathrm{P}^{3}$ biting , the results of a specimen-specific in silico (FEA) loading analysis, and an estimate of the error in the FEA, where "error" is represented by the difference between in vitro (observed) and in silico (expected) results, divided by the expected results. See Fig. S3 - Fig. S7 for site locations. Units are in microstrain $(\mu \varepsilon)$. 


\begin{tabular}{llcc|llcc}
\hline Site & Exp. & MaxPrin & MinPrin & Site & Exp. & MaxPrin & MinPrin \\
\hline 1. & In vitro & $15.00(4.36)$ & $-10.33(2.08)$ & $\mathbf{8 .}$ & In vitro & $42.33(2.08)$ & $-109.67(3.06)$ \\
& In silico & 14 & -15 & & In silico & 37 & -105 \\
& Error & $6.67 \%$ & $45.16 \%$ & & Error & $12.60 \%$ & $4.26 \%$ \\
2. & In vitro & $13.00(1.00)$ & $-11.67(0.58)$ & $\mathbf{9 .}$ & In vitro & $7.67(0.58)$ & $-2.67(2.08)$ \\
& In silico & 10 & -10 & & In silico & 8 & -4 \\
& Error & $23.08 \%$ & $14.29 \%$ & & Error & $4.35 \%$ & $50.00 \%$ \\
3. & In vitro & $3.33(0.58)$ & $-5.00(1.00)$ & $\mathbf{1 0 .}$ & In vitro & $45.33(2.08)$ & $-22.33(1.15)$ \\
& In silico & 6 & -7 & & In silico & 23 & -20 \\
& Error & $80.00 \%$ & $40.00 \%$ & & Error & $49.26 \%$ & $10.45 \%$ \\
4. & In vitro & $30.67(1.15)$ & $-36.00(0.00)$ & $\mathbf{1 1 .}$ & In vitro & $23.67(0.58)$ & $-10.67(3.06)$ \\
& In silico & 29 & -34 & & In silico & 22 & -13 \\
& Error & $5.43 \%$ & $5.56 \%$ & & Error & $7.04 \%$ & $21.88 \%$ \\
5. & In vitro & $15.00(2.00)$ & $-14.67(1.53)$ & $\mathbf{1 2 .}$ & In vitro & $108.00(2.65)$ & $-281.67(8.33)$ \\
& In silico & 19 & -12 & & In silico & 115 & -238 \\
& Error & $26.67 \%$ & $18.18 \%$ & & Error & $6.48 \%$ & $15.50 \%$ \\
6. & In vitro & $11.67(0.58)$ & $-7.33(0.58)$ & $\mathbf{1 3 .}$ & In vitro & $38.67(1.15)$ & $-22.00(1.00)$ \\
& In silico & 11 & -10 & & In silico & 39 & -17 \\
& Error & $5.71 \%$ & $36.36 \%$ & & Error & $0.86 \%$ & $22.73 \%$ \\
7. & In vitro & $42.33(1.53)$ & $-23.33(2.25)$ & $\mathbf{1 4 .}$ & In vitro & $27.67(2.08)$ & $-42.33(3.01)$ \\
& In silico & 42 & -17 & & In silico & 38 & -25 \\
& Error & $0.79 \%$ & $27.14 \%$ & & Error & $37.35 \%$ & $40.94 \%$ \\
\hline
\end{tabular}

1 


\section{Table 7 (on next page)}

Variation in strain and strain energy density in the ALL-HUM models.

Coefficients of variation for maximum principal strain (MaxPrin), minimum principal strain (MinPrin), shear strain (Shear), von Mises strain, and strain energy density (SED) at the 14 locations examined during premolar $\left(\mathrm{P}^{3}\right)$ and molar $\left(\mathrm{M}^{2}\right)$ biting in the ALL-HUM models of modern human crania. Site numbers follow Figure 4. 


\begin{tabular}{llccccc}
\hline Site & Bite & MaxPrin & MinPrin & Shear & von Mises & SED \\
\hline $\mathbf{1}$ & $\mathrm{P}^{3}$ & 56.01 & 34.39 & 28.49 & 27.88 & 59.08 \\
& $\mathrm{M}^{2}$ & 43.20 & 28.62 & 20.78 & 22.82 & 50.07 \\
$\mathbf{2}$ & $\mathrm{P}^{3}$ & 28.35 & 41.61 & 30.51 & 29.27 & 78.82 \\
& $\mathrm{M}^{2}$ & 27.61 & 44.20 & 29.50 & 29.04 & 60.38 \\
$\mathbf{3}$ & $\mathrm{P}^{3}$ & 23.83 & 26.53 & 22.94 & 22.97 & 52.39 \\
& $\mathrm{M}^{2}$ & 25.16 & 24.29 & 24.66 & 24.16 & 49.48 \\
$\mathbf{4}$ & $\mathrm{P}^{3}$ & 15.30 & 21.39 & 14.75 & 14.28 & 27.78 \\
& $\mathrm{M}^{2}$ & 34.43 & 22.83 & 22.73 & 21.46 & 36.89 \\
$\mathbf{5}$ & $\mathrm{P}^{3}$ & 14.32 & 13.06 & 12.77 & 13.24 & 26.98 \\
& $\mathrm{M}^{2}$ & 12.50 & 14.22 & 11.70 & 12.06 & 24.53 \\
$\mathbf{6}$ & $\mathrm{P}^{3}$ & 21.74 & 12.21 & 11.77 & 11.89 & 23.52 \\
& $\mathrm{M}^{2}$ & 17.43 & 13.56 & 11.13 & 12.05 & 25.11 \\
$\mathbf{7}$ & $\mathrm{P}^{3}$ & 12.53 & 8.26 & 8.09 & 7.93 & 15.97 \\
& $\mathrm{M}^{2}$ & 11.27 & 6.05 & 5.78 & 5.32 & 11.98 \\
$\mathbf{8}$ & $\mathrm{P}^{3}$ & 19.73 & 2.58 & 13.87 & 12.50 & 25.96 \\
& $\mathrm{M}^{2}$ & 20.48 & 12.04 & 12.62 & 11.88 & 23.36 \\
$\mathbf{9}$ & $\mathrm{P}^{3}$ & 20.78 & 21.84 & 18.18 & 19.30 & 39.77 \\
& $\mathrm{M}^{2}$ & 12.59 & 9.28 & 8.23 & 8.66 & 19.36 \\
$\mathbf{1 0}$ & $\mathrm{P}^{3}$ & 11.70 & 33.05 & 12.32 & 11.72 & 21.21 \\
& $\mathrm{M}^{2}$ & 35.51 & 22.16 & 25.60 & 25.86 & 50.44 \\
$\mathbf{1 1}$ & $\mathrm{P}^{3}$ & 24.44 & 37.84 & 24.15 & 21.83 & 36.54 \\
& $\mathrm{M}^{2}$ & 25.53 & 43.20 & 28.88 & 26.73 & 52.39 \\
$\mathbf{1 2}$ & $\mathrm{P}^{3}$ & 51.04 & 35.54 & 39.39 & 37.44 & 64.43 \\
& $\mathrm{M}^{2}$ & 52.66 & 34.33 & 41.78 & 40.46 & 76.44 \\
$\mathbf{1 3}$ & $\mathrm{P}^{3}$ & 28.41 & 34.42 & 26.48 & 25.60 & 51.87 \\
& $\mathrm{M}^{2}$ & 14.11 & 20.80 & 14.37 & 13.50 & 28.05 \\
$\mathbf{1 4}$ & $\mathrm{P}^{3}$ & 35.54 & 22.56 & 31.16 & 31.33 & 68.31 \\
& $\mathrm{M}^{2}$ & 39.93 & 26.73 & 35.19 & 35.33 & 80.97 \\
\hline & & & & & &
\end{tabular}

1

2 


\section{Table 8 (on next page)}

Variation in von Mises strain magnitudes: Human vs. Chimpanzee.

Comparisons of the coefficients of variation (CVs) for von Mises strain recorded in the CHIMPED human models and the chimpanzee results from Smith et al. (2015b) at each of the 14 craniofacial sites examined. Results of Fligner-Killeen tests for equal CVs between the species are also presented $(\alpha=0.05)$. Comparisons that yielded significant results are shown in bold typeface. 


\begin{tabular}{llccclcc}
\hline Site & & $\mathbf{P}^{\mathbf{3}}$ & $\mathbf{M}^{\mathbf{2}}$ & Site & & $\mathbf{P}^{\mathbf{3}}$ & $\mathbf{M}^{\mathbf{2}}$ \\
\hline $\mathbf{1}$ & CV - Human & 29.04 & 22.68 & $\mathbf{8}$ & CV - Humans & 10.14 & 12.27 \\
& CV - Chimp & 25.91 & 23.63 & & CV - Chimps & 16.54 & 25.58 \\
& p (same CV) & 0.065 & 0.141 & & p (same CV) & 0.143 & 0.130 \\
$\mathbf{2}$ & CV - Humans & 24.34 & 23.05 & $\mathbf{9}$ & CV - Humans & 14.12 & 8.03 \\
& CV - Chimps & 46.61 & 47.07 & & CV - Chimps & 25.7 & 23.58 \\
& p (same CV) & 0.122 & 0.050 & & p (same CV) & 0.069 & 0.052 \\
$\mathbf{3}$ & CV - Humans & 19.71 & 17.75 & $\mathbf{1 0}$ & CV - Humans & 8.8 & 15.46 \\
& CV - Chimps & 19.81 & 20.10 & & CV - Chimps & 17.36 & 15.30 \\
& p (same CV) & 0.386 & 0.369 & & p (same CV) & $\mathbf{0 . 0 3 9}$ & 0.290 \\
$\mathbf{4}$ & CV - Humans & 13.51 & 21.12 & $\mathbf{1 1}$ & CV - Humans & 10.6 & 14.34 \\
& CV - Chimps & 29.98 & 33.20 & & CV - Chimps & 27.76 & 28.11 \\
& p (same CV) & 0.176 & 0.359 & & p (same CV) & 0.056 & 0.100 \\
$\mathbf{5}$ & CV - Humans & 12.89 & 11.50 & $\mathbf{1 2}$ & CV - Humans & 38.05 & 38.76 \\
& CV - Chimps & 27.56 & 29.40 & & CV - Chimps & 28.23 & 43.35 \\
& p (same CV) & 0.156 & 0.060 & & p (same CV) & 0.147 & 0.396 \\
$\mathbf{6}$ & CV - Humans & 18.15 & 16.51 & $\mathbf{1 3}$ & CV - Humans & 24.54 & 10.39 \\
& CV - Chimps & 64.99 & 66.99 & & CV - Chimps & 17.95 & 17.52 \\
& p (same CV) & $\mathbf{0 . 0 2 2}$ & $\mathbf{0 . 0 2 2}$ & & p (same CV) & 0.157 & 0.207 \\
\multirow{2}{*}{7} & CV - Humans & 11.96 & 12.07 & $\mathbf{1 4}$ & CV - Humans & 22.78 & 23.11 \\
& CV - Chimps & 55.83 & 56.63 & & CV - Chimps & 51.99 & 55.84 \\
& p (same CV) & $\mathbf{0 . 0 2 2}$ & $\mathbf{0 . 0 2 2}$ & & p (same CV) & 0.222 & 0.166 \\
\hline \multirow{4}{*}{} & & & & & & &
\end{tabular}

1 


\section{Table 9 (on next page)}

Bite force production, biting efficiency, and joint reaction forces in the ALL-HUM model variants of human crania.

Bite force (BF), mechanical advantage (MA), working-side TMJ reaction force (RF-WS), and balancing-side TMJ reaction force (RF-BS) for premolar and molar biting. Five of seven ALLHUM models generated distractive (tensile) reaction forces during molar loading. Therefore, balancing side muscle forces were iteratively reduced by $5 \%$ and re-run until distractive forces were eliminated. Bite forces and TMJ reaction forces are in Newtons (N). 


\begin{tabular}{|c|c|c|c|c|c|c|c|c|c|}
\hline \multirow[t]{2}{*}{ Model } & \multirow{2}{*}{$\begin{array}{c}\text { Muscle } \\
\text { Force }\end{array}$} & \multicolumn{4}{|c|}{ Premolar Bite } & \multicolumn{4}{|c|}{ Molar Bite } \\
\hline & & BF & MA & RF-WS & RF-BS & BF & MA & RF-WS & RF-BS \\
\hline GRGL & 1118 & 441 & 0.39 & 167.42 & 349.25 & 658 & 0.59 & -11.74 & 329.79 \\
\hline GRGL $^{1}$ & 1090 & & & & & 642 & 0.59 & -1.37 & 311.18 \\
\hline GRGL $^{2}$ & 1062 & & & & & 625 & 0.59 & 8.98 & 292.58 \\
\hline BERG & 1026 & 439 & 0.43 & 147.72 & 281.55 & 663 & 0.65 & -6.98 & 249.09 \\
\hline BERG $^{1}$ & 1000 & & & & & 647 & 0.65 & 1.29 & 234.72 \\
\hline KSAN1 & 946 & 378 & 0.40 & 121.76 & 295.69 & 538 & 0.57 & -17.49 & 280.57 \\
\hline KSAN $1^{2}$ & 898 & & & & & 511 & 0.57 & 0.07 & 249.74 \\
\hline KSAN2 & 791 & 333 & 0.42 & 106.83 & 240.30 & 496 & 0.63 & -18.86 & 222.80 \\
\hline KSAN2 ${ }^{2}$ & 751 & & & & & 471 & 0.63 & -4.26 & 197.88 \\
\hline $\mathrm{KSAN} 2^{3}$ & 732 & & & & & 459 & 0.63 & 3.04 & 185.41 \\
\hline MALP & 842 & 344 & 0.41 & 131.09 & 277.66 & 537 & 0.64 & -19.85 & 274.49 \\
\hline MALP $^{2}$ & 800 & & & & & 510 & 0.64 & -0.99 & 242.97 \\
\hline TIGA & 1246 & 507 & 0.41 & 187.96 & 373.24 & 756 & 0.61 & 13.68 & 336.84 \\
\hline WAFR & 1006 & 341 & 0.34 & 149.36 & 298.77 & 529 & 0.53 & 12.64 & 273.79 \\
\hline
\end{tabular}

$1{ }^{1}$ Model re-run using muscle forces reduced by $5 \%$ on the balancing side.

$2{ }^{2}$ Model re-run using muscle forces reduced by $10 \%$ on the balancing side.

$3 \quad{ }^{3}$ Model re-run using muscle forces reduced by $15 \%$ on the balancing side. 


\section{Table $\mathbf{1 0}$ (on next page)}

Von Mises strain magnitudes: Human vs. Chimpanzee.

Results of pairwise comparisons (Mann-Whitney U-test) of von Mises strain magnitudes at the 14 locations examined between CHIMPED variants of human FEMs and data on chimpanzees from Smith et al. (2015b). Because of small sample sizes, the "exact" variant of $p$ is reported (Mundry and Fischer, 1998). Comparisons that yielded significant results following HolmBonferroni correction are shown in bold typeface. When significant, humans were found to exhibit the higher average value, with the exception of locations 13 and 14, where humans were found to exhibit significantly lower strain magnitudes. 


\begin{tabular}{llccr}
\hline Site & Bite & U & Z & Exact p \\
\hline 1. Dorsal interorbital & Premolar & 9 & -1.65 & 0.0967 \\
& Molar & 10 & -1.50 & 0.1265 \\
2. Working dorsal orbital & Premolar & 0 & -2.93 & $\mathbf{0 . 0 0 1 2}$ \\
& Molar & 0 & -2.93 & $\mathbf{0 . 0 0 1 2}$ \\
3. Balancing dorsal orbital & Premolar & 4 & -2.36 & $0.0140^{1}$ \\
& Molar & 7 & -1.93 & 0.0513 \\
4. Working postorbital bar & Premolar & 0 & -2.93 & $\mathbf{0 . 0 0 1 2}$ \\
& Molar & 1 & -2.79 & $\mathbf{0 . 0 0 2 3}$ \\
5. Balancing postorbital bar & Premolar & 0 & -2.93 & $\mathbf{0 . 0 0 1 2}$ \\
& Molar & 0 & -2.93 & $\mathbf{0 . 0 0 1 2}$ \\
6. Working zygomatic arch & Premolar & 14 & -0.93 & 0.3660 \\
& Molar & 14 & -0.93 & 0.3660 \\
7. Balancing zygomatic arch & Premolar & 14 & -0.93 & 0.3660 \\
& Molar & 14 & -0.93 & 0.3660 \\
8. Working zygomatic root & Premolar & 0 & -2.93 & $\mathbf{0 . 0 0 1 2}$ \\
& Molar & 0 & -2.93 & $\mathbf{0 . 0 0 1 2}$ \\
9. Balancing zygo root & Premolar & 18 & -0.36 & 0.7308 \\
& Molar & 11 & -1.36 & 0.1807 \\
10. Working infraorbital & Premolar & 2 & -2.64 & $\mathbf{0 . 0 0 4 7}$ \\
& Molar & 7.5 & -1.86 & 0.0565 \\
11. Balancing infraorbital & Premolar & 6 & -2.07 & 0.0350 \\
& Molar & 12 & -1.21 & 0.2343 \\
12. Working nasal margin & Premolar & 0 & -2.93 & $\mathbf{0 . 0 0 1 2}$ \\
& Molar & 1 & -2.79 & $\mathbf{0 . 0 0 2 3}$ \\
13. Working zygomatic body & Premolar & 0 & -2.93 & $\mathbf{0 . 0 0 1 2}$ \\
& Molar & 1 & -2.79 & $\mathbf{0 . 0 0 2 3}$ \\
14. Balancing zygomatic body & Premolar & 0.5 & -2.86 & $\mathbf{0 . 0 0 1 7}$ \\
& Molar & 1 & -2.79 & $\mathbf{0 . 0 0 2 3}$ \\
\hline
\end{tabular}




\section{Table $\mathbf{1 1}$ (on next page)}

Bite force production, biting efficiency, and joint reaction forces in the CHIMPED model variants of human crania.

Bite force (BF), mechanical advantage (MA), working-side temporomandibular joint reaction force (RF-WS), and balancing-side temporomandibular joint reaction force (RF-BS) for premolar and molar biting. All seven CHIMPED models generated highly distractive (tensile) reaction forces during molar loading that would have increased the chances of joint dislocation and/or injury. Therefore, balancing side muscle forces were iteratively reduced by $5 \%$ and re-run until distractive forces were eliminated. Bite forces and TMJ reaction forces are in Newtons (N). 


\begin{tabular}{|c|c|c|c|c|c|c|c|c|c|}
\hline \multirow[t]{2}{*}{ Model } & \multirow{2}{*}{$\begin{array}{l}\text { Muscle } \\
\text { Force }\end{array}$} & \multicolumn{4}{|c|}{ Premolar Bite } & \multicolumn{4}{|c|}{ Molar Bite } \\
\hline & & BF & MA & RF-WS & RF-BS & BF & MA & RF-WS & RF-BS \\
\hline$\overline{\text { GRGL }}$ & 3965 & 1724 & 0.43 & 499.82 & 1189.57 & 2570 & 0.65 & -208.16 & 1113.51 \\
\hline GRGL $^{1}$ & 3569 & & & & & 2316 & 0.65 & -31.26 & 841.64 \\
\hline GRGL $^{2}$ & 3469 & & & & & 2252 & 0.65 & 12.96 & 773.68 \\
\hline BERG & 3637 & 1720 & 0.47 & 405.08 & 935.03 & 2599 & 0.71 & -185.65 & 819.81 \\
\hline $\mathrm{BERG}^{2}$ & 3183 & & & & & 2277 & 0.71 & -6.72 & 560.17 \\
\hline $\mathrm{BERG}^{3}$ & 3092 & & & & & 2213 & 0.71 & 29.07 & 508.24 \\
\hline KSAN1 & 3353 & 1462 & 0.44 & 343.26 & 1030.37 & 2080 & 0.62 & -187.95 & 975.38 \\
\hline KSAN $1^{2}$ & 2934 & & & & & 1822 & 0.62 & -0.30 & 687.33 \\
\hline KSAN $1^{3}$ & 2850 & & & & & 1771 & 0.62 & 37.23 & 629.72 \\
\hline KSAN2 & 2804 & 1272 & 0.45 & 311.70 & 821.79 & 1895 & 0.68 & -163.75 & 757.22 \\
\hline KSAN2 ${ }^{2}$ & 2454 & & & & & 1658 & 0.68 & -11.46 & 529.80 \\
\hline $\mathrm{KSAN} 2^{3}$ & 2384 & & & & & 1610 & 0.68 & 18.99 & 484.32 \\
\hline MALP & 2986 & 1358 & 0.45 & 384.41 & 966.38 & 2118 & 0.71 & -203.31 & 963.66 \\
\hline MALP $^{2}$ & 2613 & & & & & 1851 & 0.71 & -2.01 & 667.11 \\
\hline MALP 3 & 2538 & & & & & 1797 & 0.71 & 38.25 & 607.81 \\
\hline TIGA & 4418 & 1941 & 0.44 & 564.13 & 1288.46 & 2896 & 0.66 & -107.59 & 1143.16 \\
\hline TIGA $^{4}$ & 4197 & & & & & 2750 & 0.66 & -13.27 & 997.33 \\
\hline TIGA $^{5}$ & 4086 & & & & & 2678 & 0.66 & 33.89 & 924.42 \\
\hline WAFR & 3567 & 1383 & 0.39 & 489.34 & 1103.22 & 2146 & 0.60 & -61.09 & 1006.50 \\
\hline WAFR $^{6}$ & 3478 & & & & & 2091 & 0.60 & -24.01 & 946.69 \\
\hline WAFR $^{4}$ & 3389 & & & & & 2036 & 0.60 & 13.07 & 886.88 \\
\hline
\end{tabular}

$1{ }^{1}$ Model re-run using muscle forces reduced by $20 \%$ on the balancing side.

$2{ }^{2}$ Model re-run using muscle forces reduced by $25 \%$ on the balancing side.

$3{ }^{3}$ Model re-run using muscle forces reduced by $30 \%$ on the balancing side.

$4{ }^{4}$ Model re-run using muscle forces reduced by $10 \%$ on the balancing side.

$5 \quad{ }^{5}$ Model re-run using muscle forces reduced by $15 \%$ on the balancing side.

$6{ }^{6}$ Model re-run using muscle forces reduced by $5 \%$ on the balancing side. 


\section{1}

The constrained lever model of jaw biomechanics.

During biting, the bite point (b) and the temporomandibular joints on the working side (ws) and balancing side (bs) form a "triangle of support" that changes shape when biting on different teeth. During a premolar bite (A), the resultant vector of the jaw adductor muscles (v) passes through the triangle, producing compression (green circles) at all three points. However, during some molar bites (B), the vector falls outside the triangle when the muscles are being recruited equally on both sides of the head, producing compression at the bite point and bs joint, but distraction (red circle) at the ws joint. The recruitment of the balancing side muscles must be lessened in order to eliminate this distraction, thereby causing the vector to shift its position towards the working side and back into the triangle (yellow arrow).
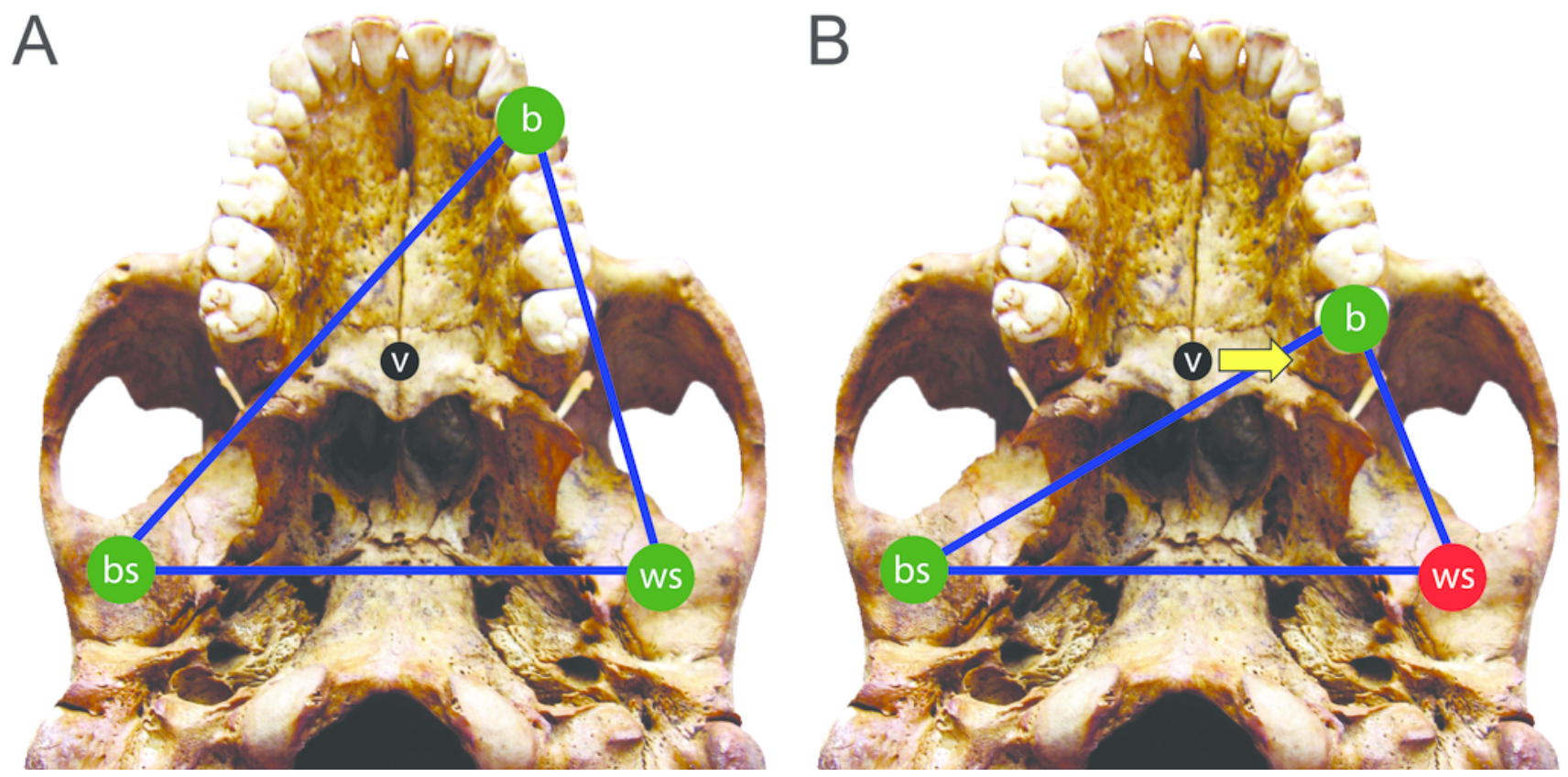


\section{2}

Principal component analysis (PCA) of human craniofacial shape variation.

Panels show (A) PC1 by PC2, (B) PC1 by PC3, and (C) wireframes illustrating craniofacial shape change associated with the first three principal components in right lateral, superior, and frontal views. The left and right columns of wireframes represent the negative and positive ends of each component, respectively, scaled to their respective axes. The 10 unique landmarks with the highest loadings for each component are highlighted using a red ellipse on the midline and right side. A single ellipse was used to circle multiple landmarks if they were located close together. Shape differences toward the positive end of PC 1 include: a vertically shorter face with a more projecting brow ridge, a longer and more projecting palate, a more vertical frontal bone that is narrower at pterion, a vault that is expanded posteriorly, and a lower temporal line at stephanion. Shape differences toward the positive end of PC 2 include: a longer cranium with a wider frontal bone, a vault that is angled more postero-inferiorly, wider orbits and a superiorly shifted nasal aperture, and an anteroposteriorly shorter temporal bone. Shape differences toward the positive end of PC 3 include: higher temporal lines at stephanion, a shorter and more orthognathic subnasal region with a less projecting palate, a more inferiorly positioned temporomandibular joint, and a more inferiorly positioned midline cranial base. 

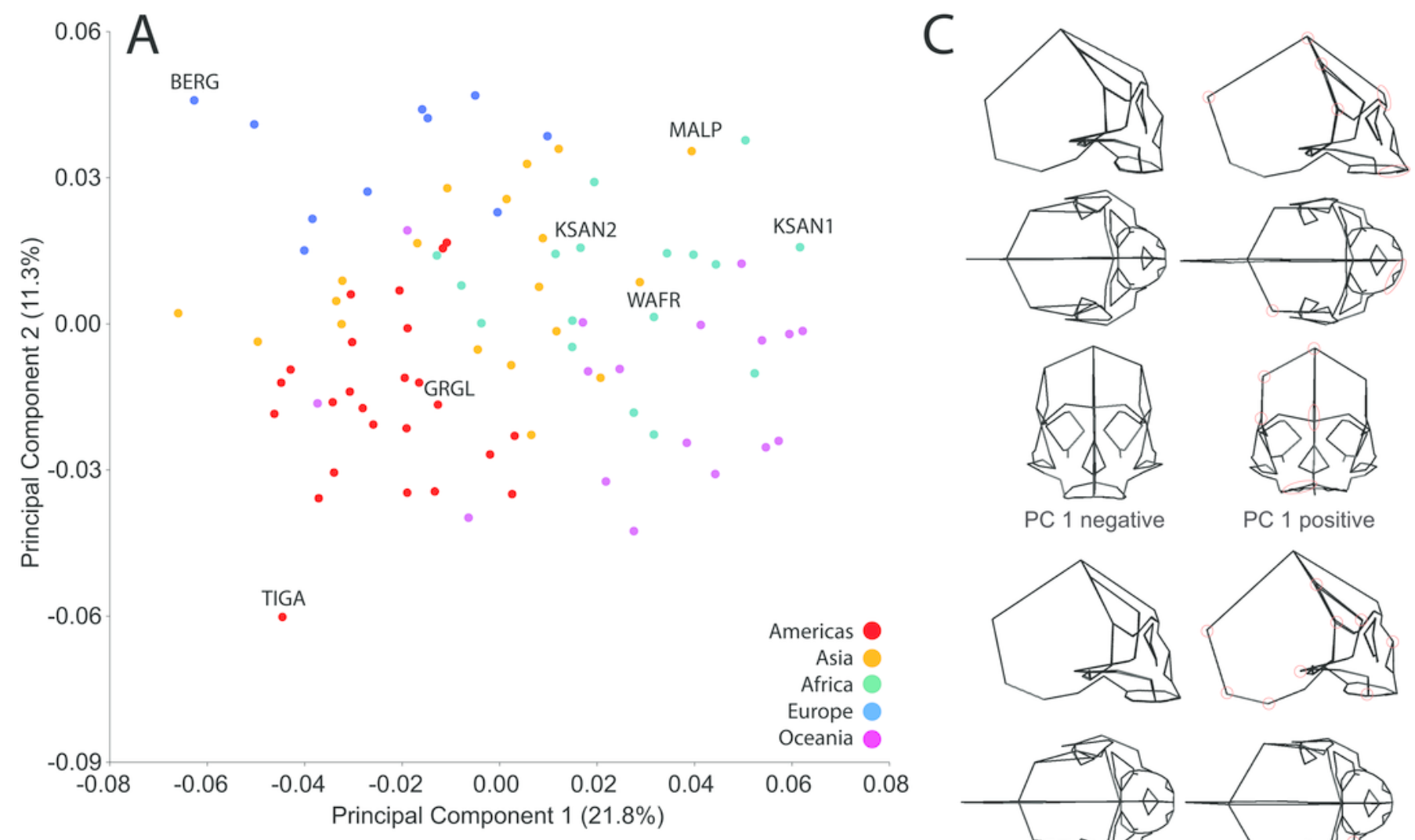

PC 1 negative

PC 1 positive
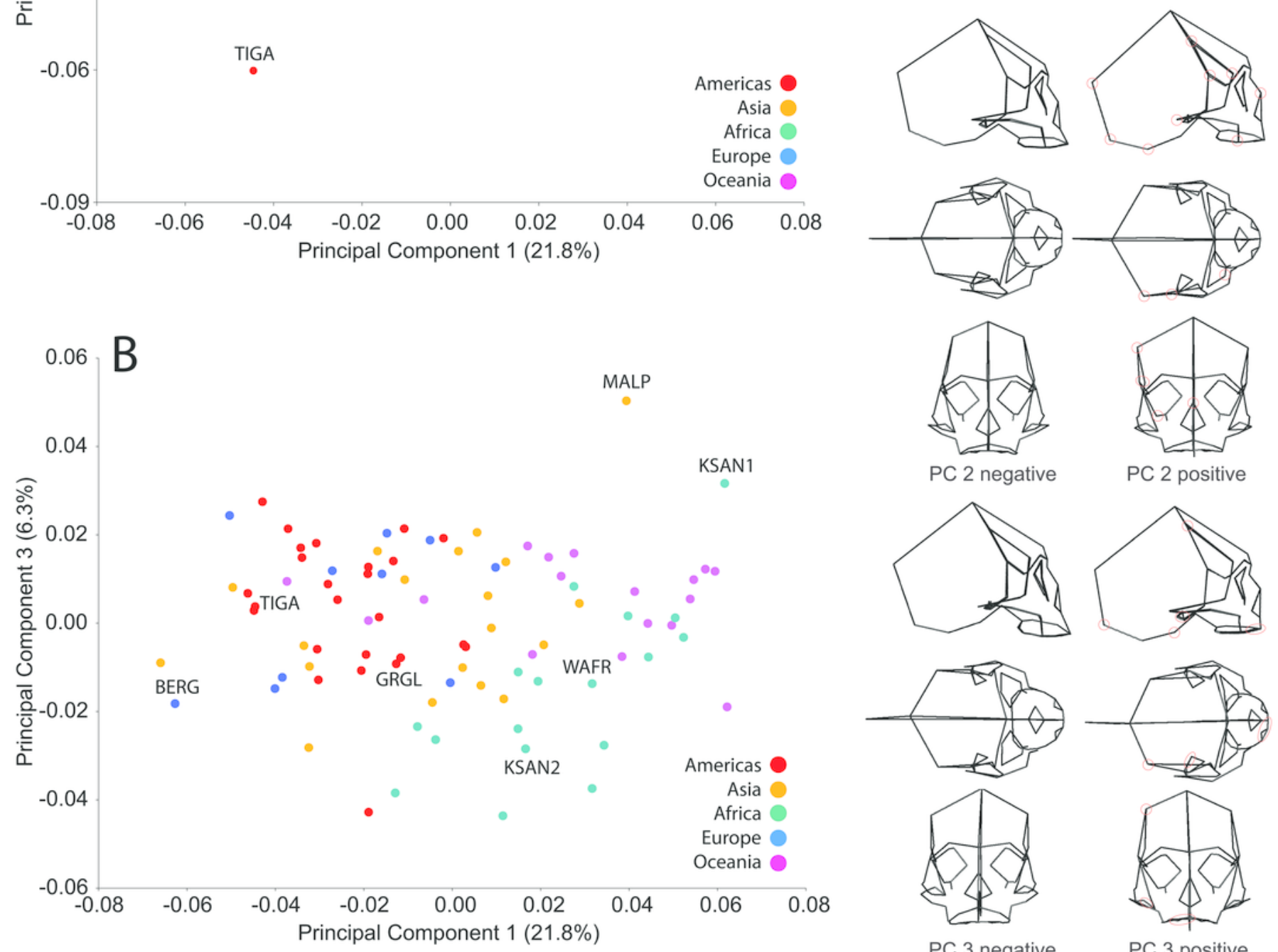

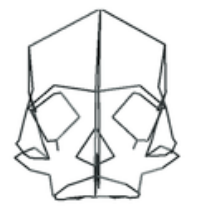

PC 3 negative

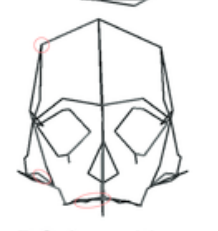

PC 3 positive 
3

Human models analyzed in the current study.

Models include one "average" cranium, GRGL (A), and six "extreme" specimens that differ notably in shape, BERG (B), KSAN1 (C), KSAN2 (D), MALP (E), TIGA (F), and WAFR (G). 

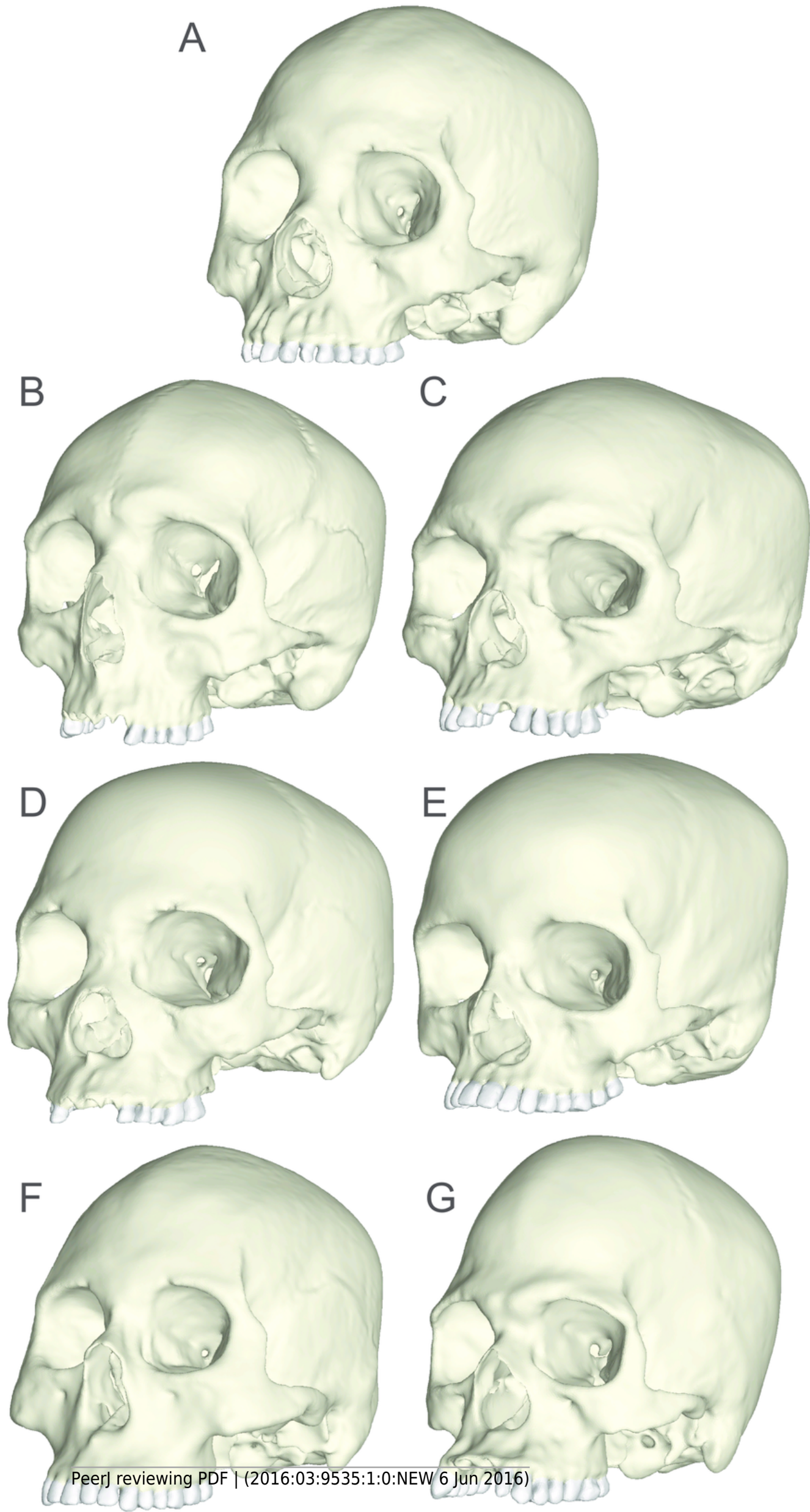
4

Key to locations where strains were sampled in finite element models.

Strain data were collected from ALL-HUM and CHIMPED variants of human FEMs from 14 craniofacial sites, following Smith et al. (2015a,b).

Dorsal Interorbital Working Dorsal Orbital Balancing Dorsal Orbital Working Postorbital Bar Balancing Postorbital Bar Working Zygomatic Arch Balancing Zygomatic Arch Working Zygomatic Root Balancing Zygomatic Root Working Infraorbital Balancing Infraorbital Working Nasal Margin Working Zygomatic Body Balancing Zygomatic Body

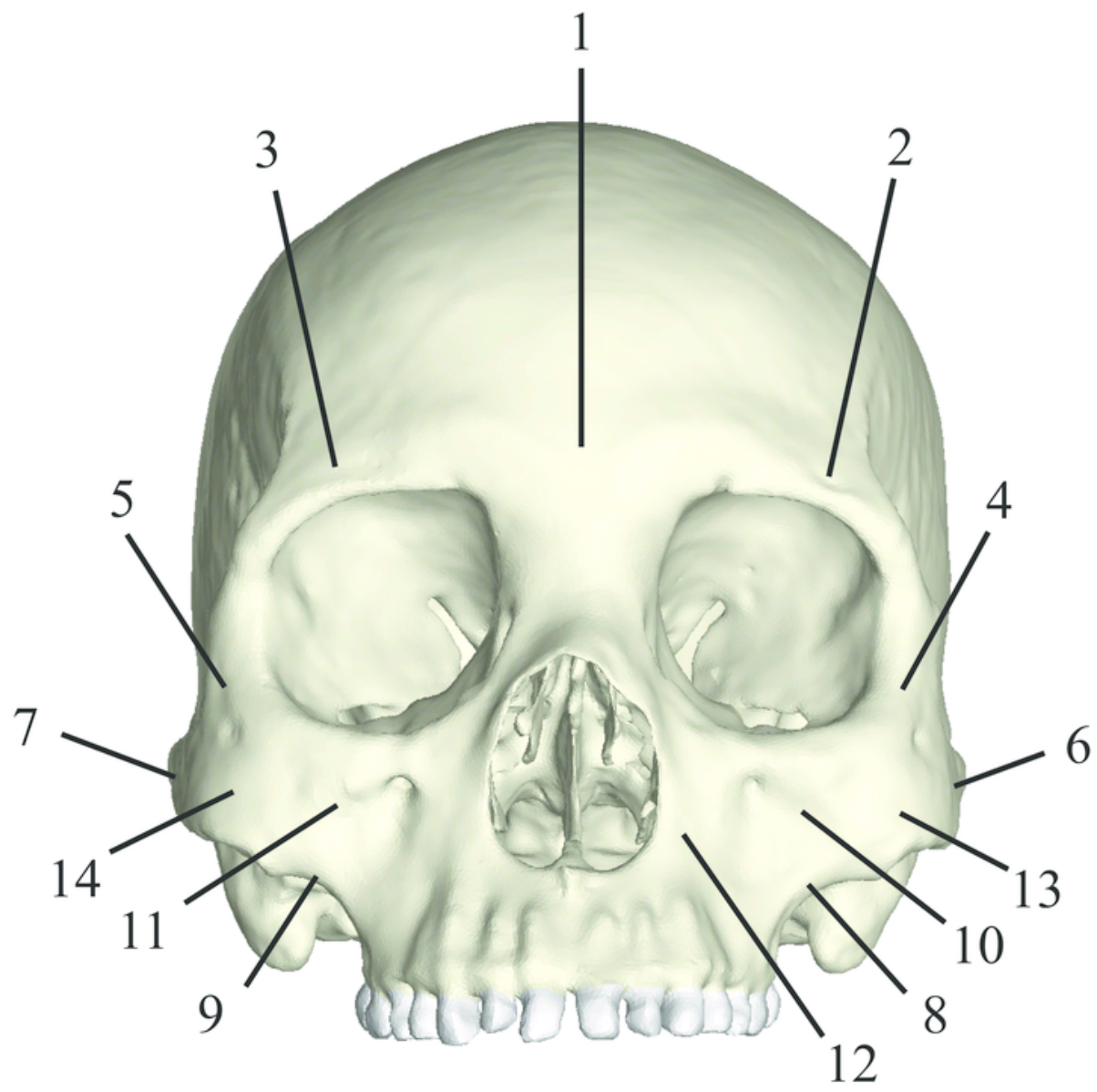




\section{5}

Strain and SED generated by the ALL-HUM models.

Box-and-whisker plots show the minimum, first quartile, median, third quartile, and maximum for strain and SED magnitudes (y-axis) generated by the ALL-HUM models at the 14 sampled locations ( $\mathrm{X}$-axis) during premolar $\left(\mathrm{P}^{3}\right)$ and molar $\left(\mathrm{M}^{2}\right)$ biting. Site numbers follow Fig. 4. 

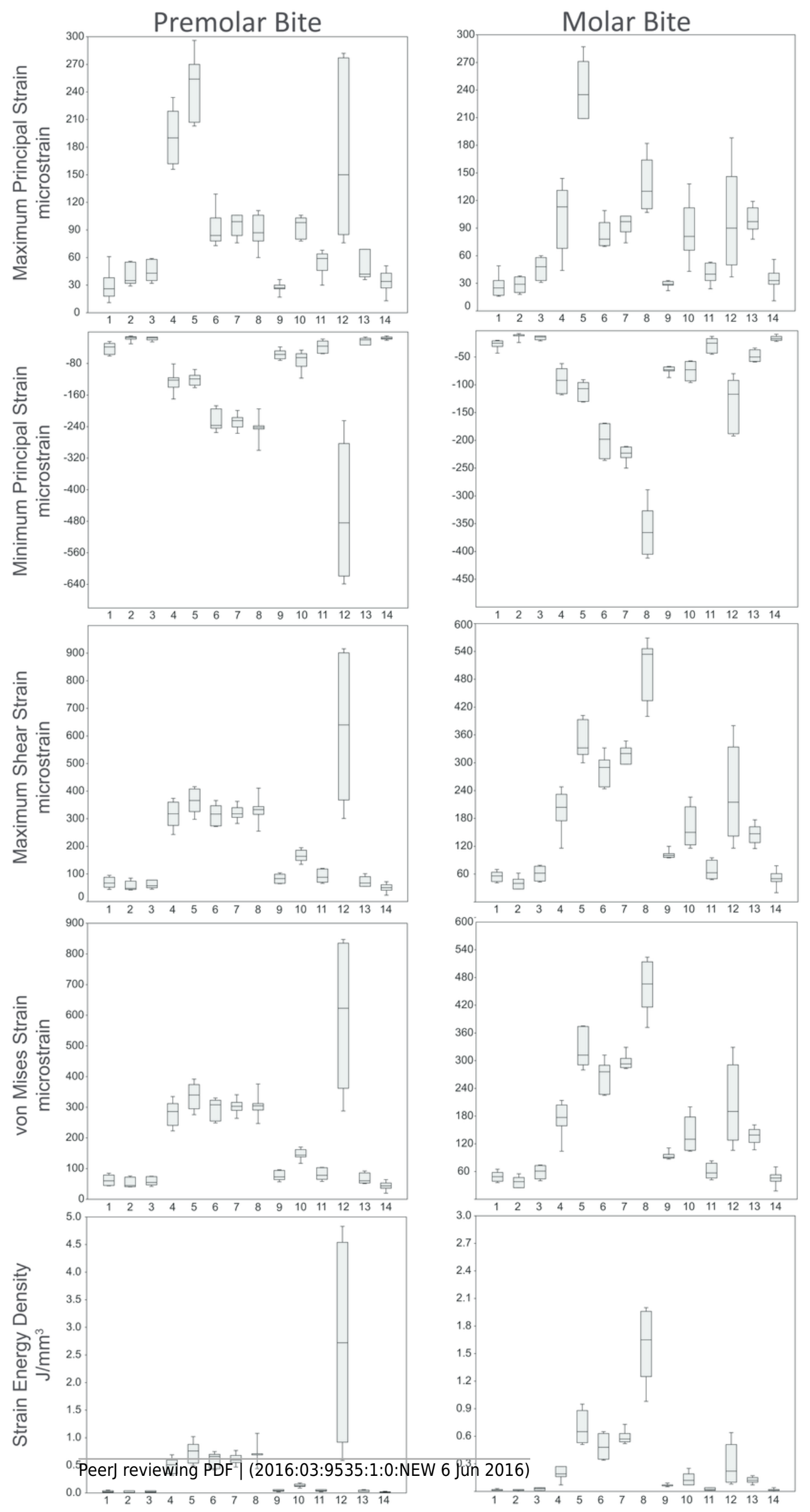
6

Strain mode in the ALL-HUM models.

Distribution of strain mode (log of ratio of maximum to minimum principal strain, $y$-axis) plotted by location (x-axis) in the ALL-HUM models. Plots show (A) premolar $\left(\mathrm{P}^{3}\right)$ and (B) molar $\left(\mathrm{M}^{2}\right)$ biting. Logging the data listed in Tables S2 and S3 centers strain mode data around zero. Values above zero indicate mainly tension, while values below zero indicate mainly compression. Site numbers follow Fig. 4. 

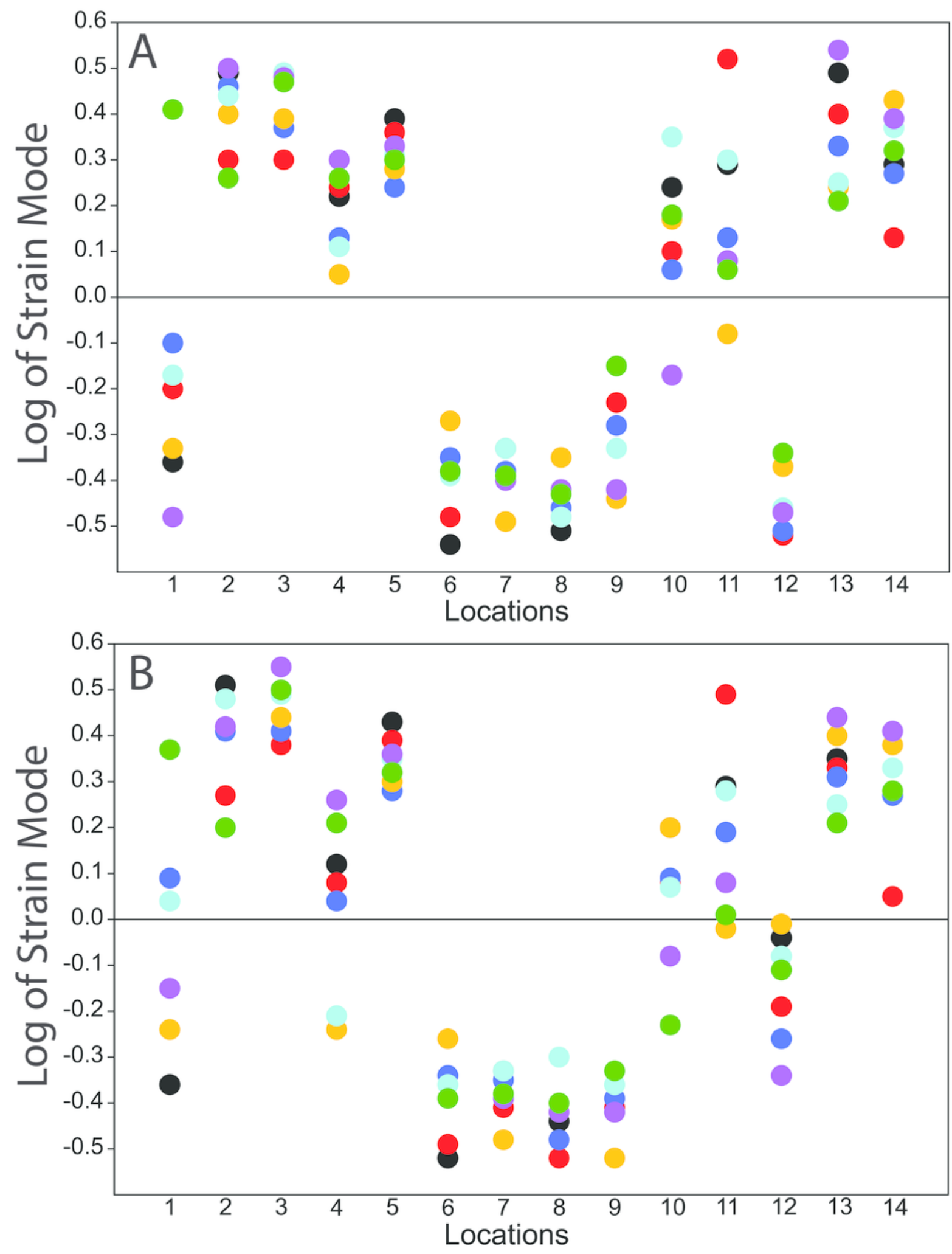

GRGL

- BERG

KSAN1

KSAN2

MALP

TIGA

WAFR 


\section{7}

Strain distributions in the ALL-HUM models: $\mathrm{P}^{3}$ biting.

Color maps of strain distributions in the ALL-HUM variants of "extreme" and "average" modern human cranial FEMs during premolar $\left(\mathrm{P}^{3}\right)$ biting. Scales are set to range from -150 $150 \mu \varepsilon$ for both maximum principal strain (MaxPrin) and minimum principal strain (MinPrin), from 0 - $300 \mu \varepsilon$ for both maximum shear strain (Shear) and von Mises strain (von Mises), and from $0-0.5 \mathrm{~J} / \mathrm{mm}^{3}$ for strain energy density (SED). White regions exceed scale. Models are shown at the same height. 


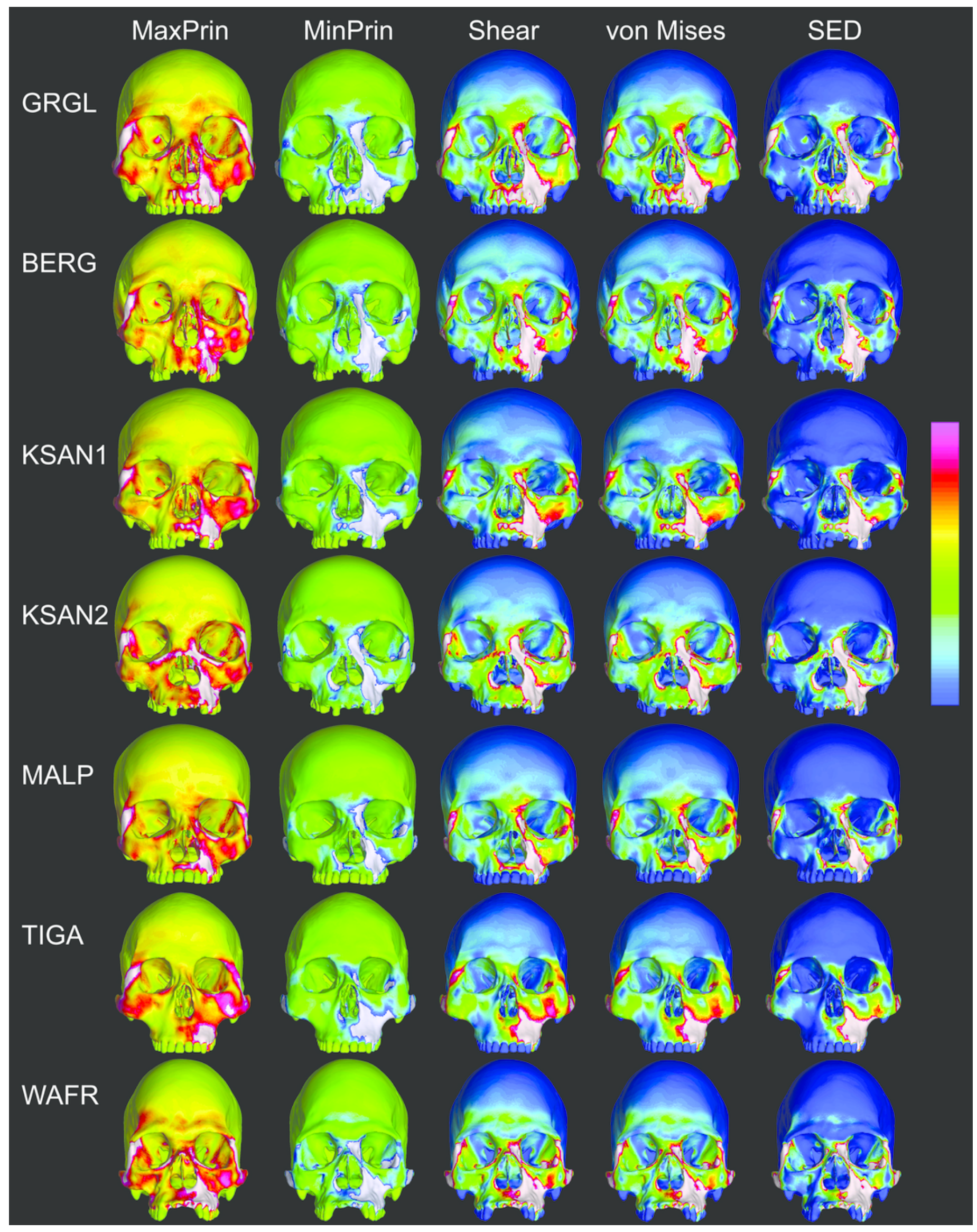


8

Strain distributions in the ALL-HUM models: $\mathrm{M}^{2}$ biting.

Color maps of strain distributions in the ALL-HUM variants of "extreme" and "average" modern human cranial FEMs during molar ( $\left.\mathrm{M}^{2}\right)$ biting. Scales are set to range from -150 - 150 $\mu \varepsilon$ for both maximum principal strain (MaxPrin) and minimum principal strain (MinPrin), from 0 - $300 \mu \varepsilon$ for both maximum shear strain (Shear) and von Mises strain (von Mises), and from $0-0.5 \mathrm{~J} / \mathrm{mm}^{3}$ for strain energy density (SED). White regions exceed scale. Models are shown at the same height. 


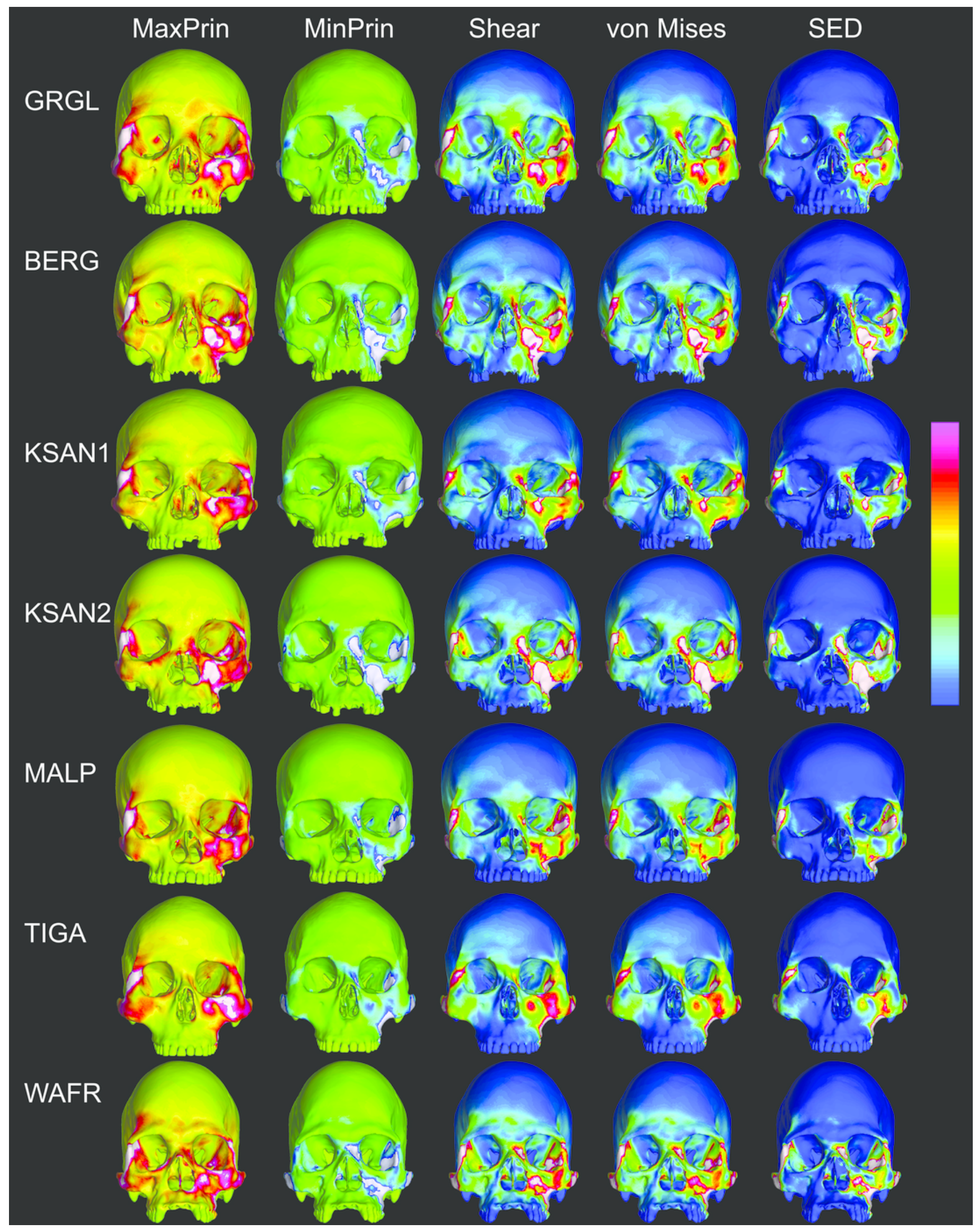


9

Relative strain distributions.

Color maps of "relative" maximum (MaxPrin) and minimum (MinPrin) principal strains in the CHIMPED model variants during premolar $\left(\mathrm{P}^{3}\right)$ and molar $\left(\mathrm{M}^{2}\right)$ biting. The scales range from $-\overline{\mathrm{X}}$ to $\bar{x}$, where $\bar{x}$ differs in each image as follows: $P^{3}$, MaxPrin/MinPrin: GRGL, 612/644; BERG, 500/534; KSAN1, 508/603; KSAN2, 593/724; MALP, 520/610; TIGA, 455/498; WAFR, 672/742; M², MaxPrin/MinPrin: GRGL, 505/546; BERG, 468/525; KSAN1, 441/473; KSAN2, 505/546; MALP, 433/458; TIGA, 419/420; WAFR, 530/553. White regions exceed scale. 


\section{Premolar Bite} MaxPrin

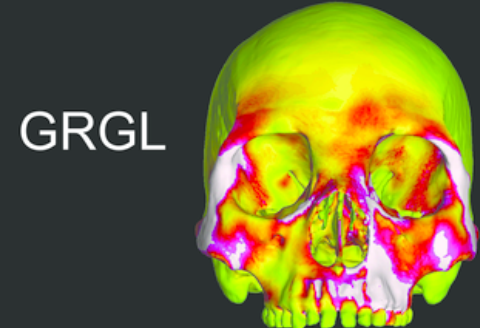

BERG
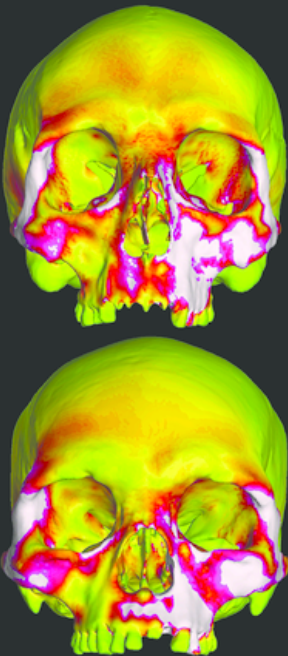

KSAN2

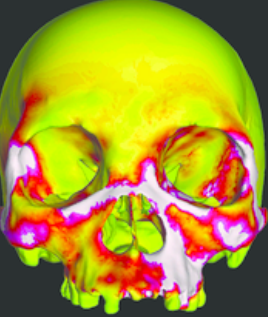

MALP

TIGA
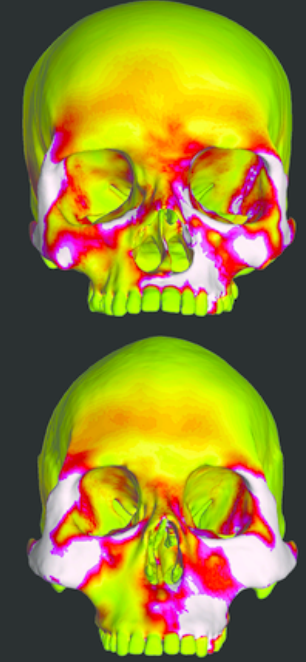

WAFR

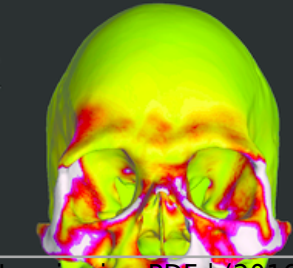

lewing PDF (2
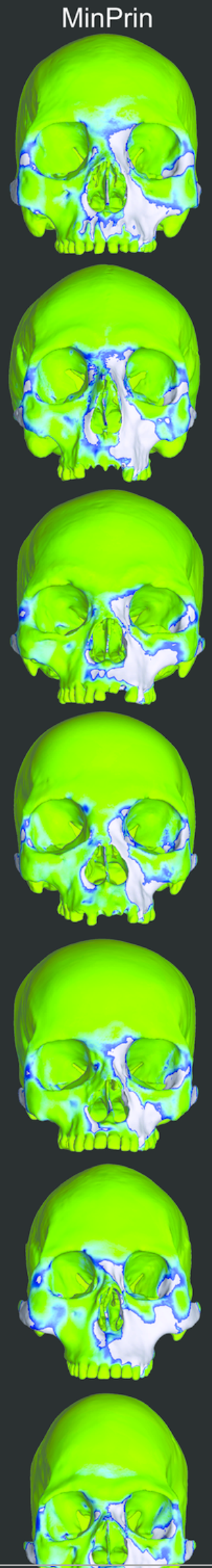
1:0:NEW 6 JU

\section{Molar Bite}
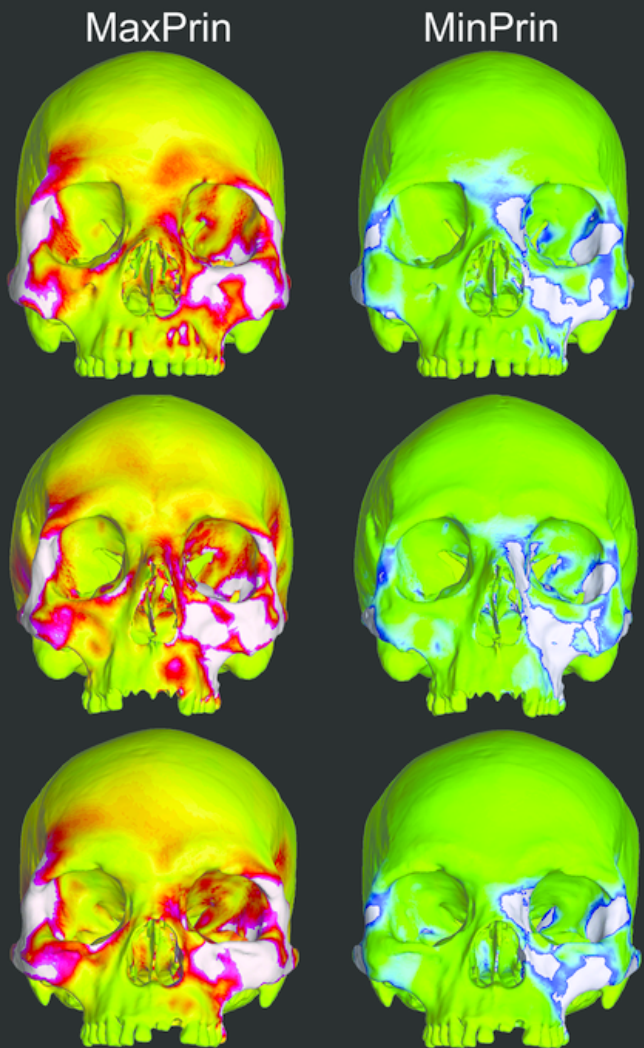

$\bar{X} \mu \varepsilon$
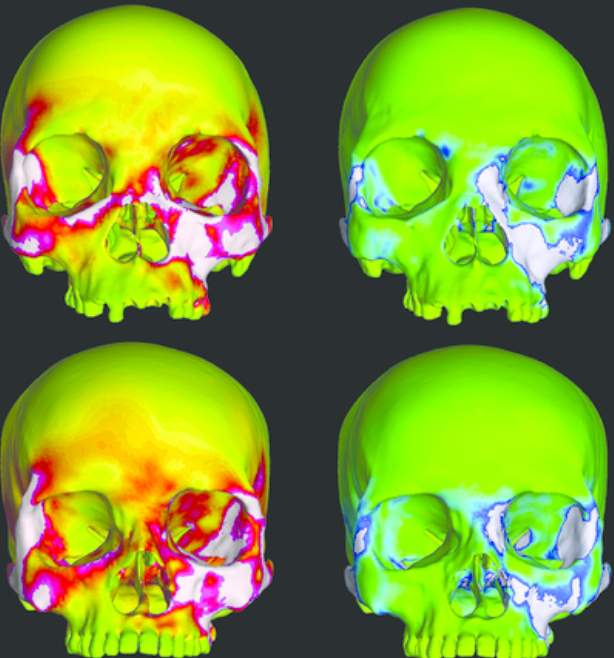

$-\bar{X} \mu \varepsilon$
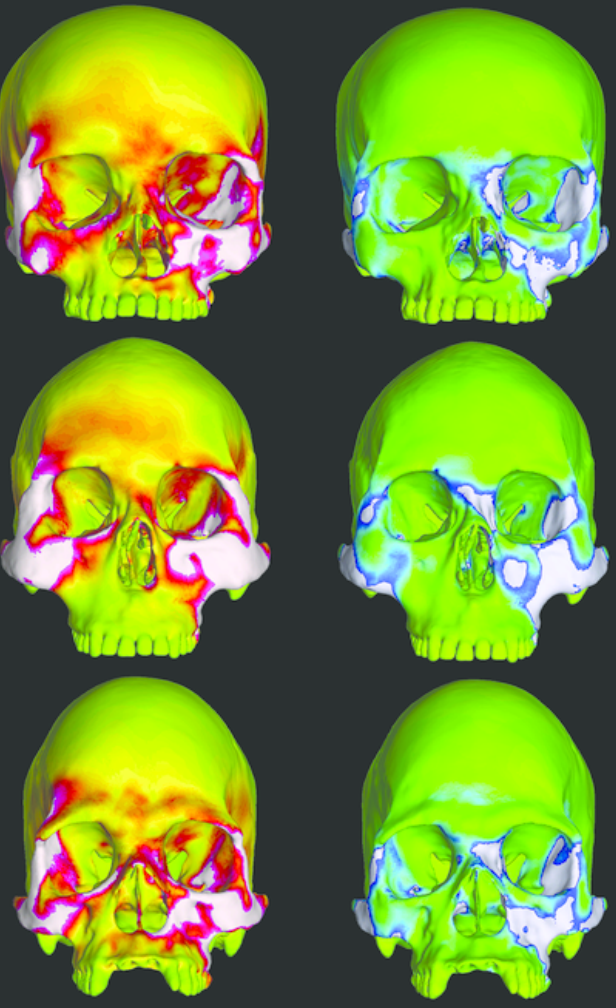
10

Line plots of von Mises microstrain generated during simulated biting in finite element models of humans and chimpanzees.

Strain data correspond to (A) left premolar $\left(\mathrm{P}^{3}\right)$ and $(\mathbf{B})$ left molar $\left(\mathrm{M}^{2}\right)$ biting, recorded from 14 homologous locations in the CHIMPED variants of "extreme" and "average" modern human cranial FEMs. The gray region brackets the range of variation observed for chimpanzees by Smith et al. (2015b). 


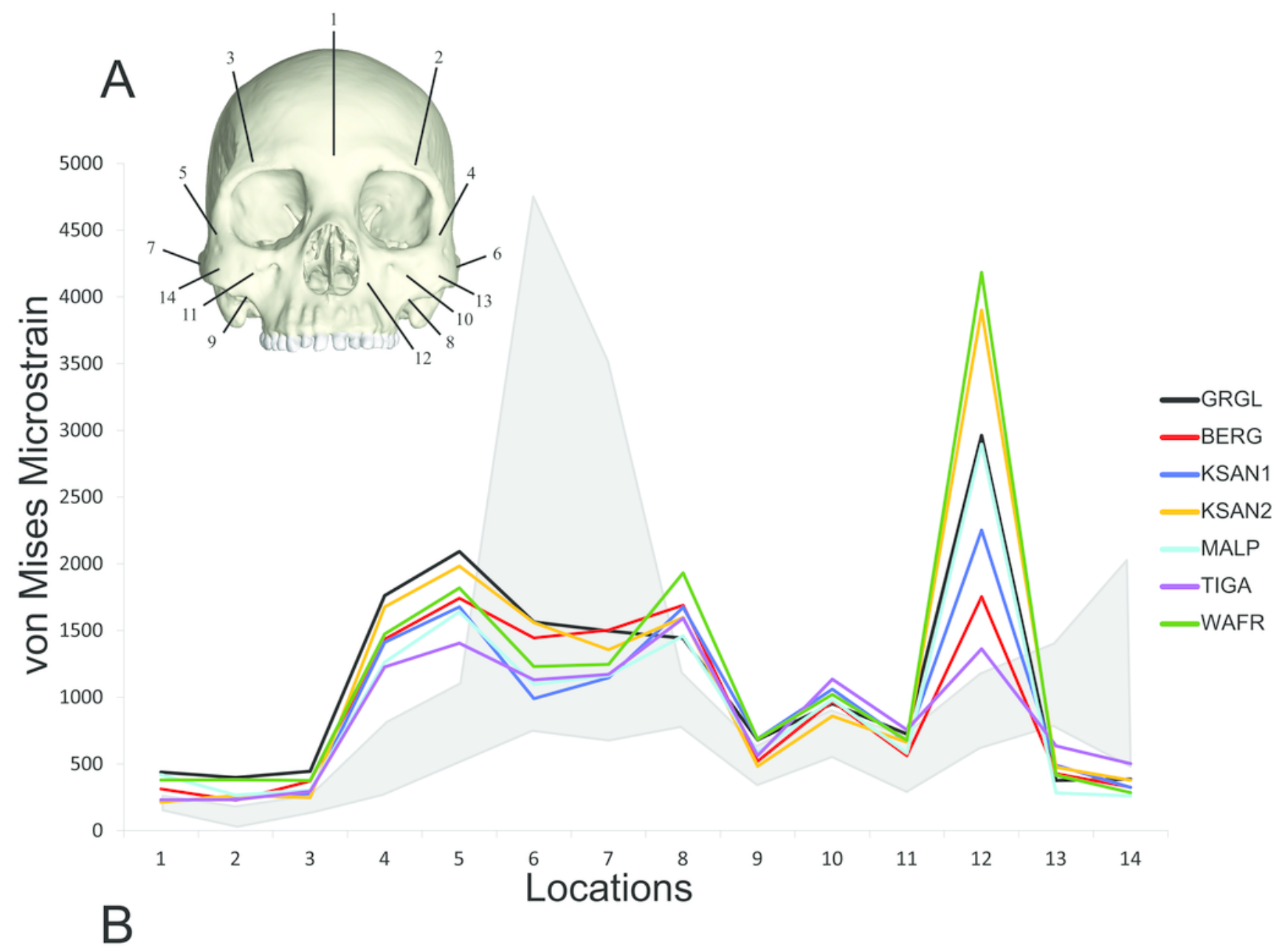

5000

4500

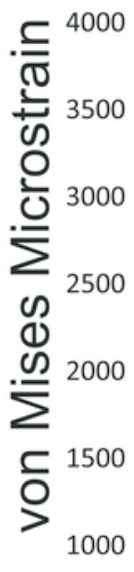

500

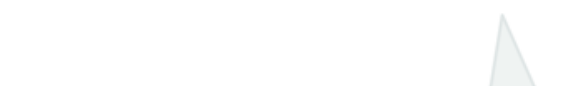


11

Biting efficiency: humans vs. chimpanzees.

Box-and-whisker plots show the minimum, first quartile, median, third quartile, and maximum biting efficiency, as quantified using the mechanical advantage (MA), in the CHIMPED variants of human cranial FEMs vs. chimpanzees at $(\mathbf{A})$ premolar $\left(\mathrm{P}^{3}\right)$ and $(\mathbf{B})$ molar $\left(M^{2}\right)$ bite points. Chimpanzee data is from Smith et al. (2015b).
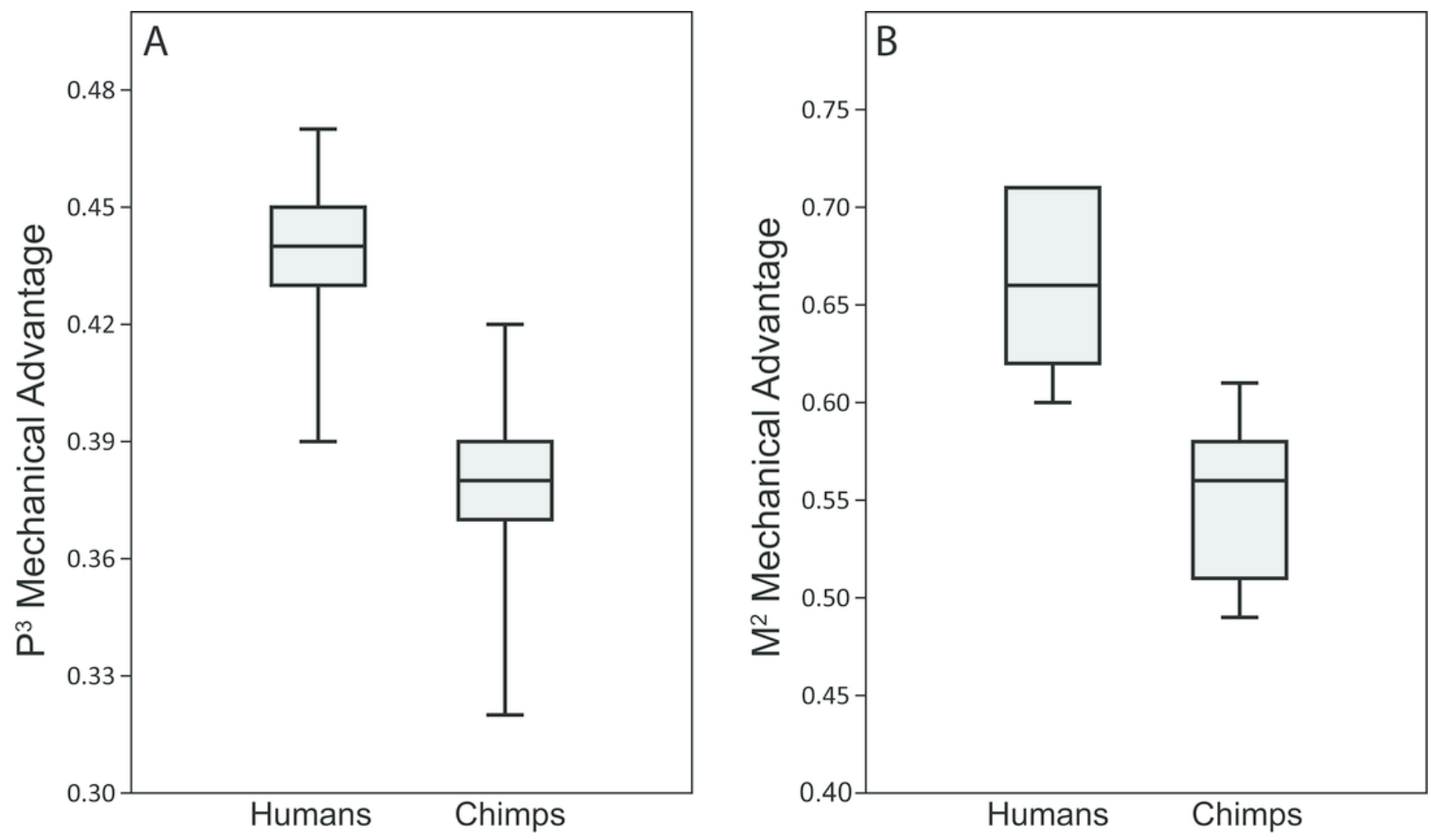